\title{
Measuring the Electron-Phonon Interaction in Two-Dimensional Superconductors with He-Atom Scattering
}

\author{
Giorgio Benedek ${ }^{1,2}{ }^{\oplus}$, Joseph R. Manson ${ }^{2,3}$, Salvador Miret-Artés ${ }^{2,4}{ }^{(0)}$, Adrian Ruckhofer ${ }^{5}$, \\ Wolfgang E. Ernst ${ }^{5}\left(\mathbb{D}\right.$, Anton Tamtögl ${ }^{5, *(1)}$ and Jan Peter Toennies ${ }^{6}$ \\ 1 Dipartimento di Scienza dei Materiali, Università di Milano-Bicocca, Via R. Cozzi 55, 20185 Milano, Italy; \\ giorgio.benedek@unimib.it \\ 2 Donostia International Physics Center (DIPC), Paseo M. de Lardizabal 4, 20018 Donostia/San Sebastián, \\ Basque Country, Spain; jmanson@clemson.edu (J.R.M.); s.miret@iff.csic.es (S.M.-A.) \\ 3 Department of Physics and Astronomy, Clemson University, Clemson, SC 29630, USA \\ 4 Institute of Fundamental Physics, Spanish Research Council, 28006 Madrid, Spain \\ 5 Institute of Experimental Physics, Graz University of Technology, 8010 Graz, Austria; \\ ruckhofer@tugraz.at (A.R.); wolfgang.ernst@tugraz.at (W.E.E.) \\ 6 Max-Planck-Institut für Dynamik und Selbstorganisation, Am Fassberg 17, 37077 Göttingen, Germany; \\ jtoenni@gwdg.de \\ * Correspondence: tamtoegl@tugraz.at
}

Received: 27 October 2020; Accepted: 27 November 2020; Published: 3 December 2020; Corrected: 16 December 2021

\begin{abstract}
Helium-atom scattering (HAS) spectroscopy from conducting surfaces has been shown to provide direct information on the electron-phonon interaction, more specifically the mass-enhancement factor $\lambda$ from the temperature dependence of the Debye-Waller exponent, and the mode-selected electron-phonon coupling constants $\lambda_{\mathbf{Q} v}$ from the inelastic HAS intensities from individual surface phonons. The recent applications of the method to superconducting ultra-thin films, quasi-1D high-index surfaces, and layered transition-metal and topological pnictogen chalcogenides are briefly reviewed.
\end{abstract}

Keywords: electron-phonon interaction; superconductivity; topological insulator; topological materials; transition metal dichalcogenide; charge density wave; helium atom scattering

\section{Introduction}

Helium-atom scattering (HAS) from a conducting surface can exchange energy and momentum with the lattice vibrations of the surface exclusively via the surface charge-density oscillations produced by the atomic motion, i.e., via electron-phonon (e-ph) interaction. Although this mechanism has been suggested since the early days of HAS spectroscopy [1], as a consequence of the discovery by HAS of the ubiquitous anomalous longitudinal surface resonance at metal surfaces [2-5], only more recent theoretical studies based on density functional perturbation theory (DFPT) [6,7] proved that the inelastic HAS intensities from surface phonons are directly proportional to their specific e-ph coupling constants $\lambda_{\mathbf{Q}}$, inaugurating what has been termed as mode-lambda spectroscopy.

An interesting aspect of the e-ph mechanism is that subsurface phonons that produce a modulation of the surface electron density can also be detected by HAS, the detection depth being equal to the range of the e-ph interaction. Thus, a surface probe such as He atoms with incident energies in the range of tens of $\mathrm{meV}$ that only tickle the surface where the electron density is $\sim 10^{-4}$ a.u. [8], can even measure the dispersion curves of phonons propagating at several atomic planes beneath the surface (quantum sonar effect), e.g., at the interface of ultra-thin metal films with the substrate, provided the e-ph coupling is sufficiently strong $[7,9]$. 
A natural consequence of this mechanism is that the thermal mean-square distortion of the surface charge-density profile, providing the Debye-Waller attenuation of the specular intensity with increasing temperature, is proportional to the mean-square phonon displacement via the total e-ph coupling, represented by the mass-enhancement factor $\lambda$. This permits the direct derivation of $\lambda$ from the temperature dependence of HAS reflectivity for any conducting surface, as demonstrated in a recent series of papers devoted to metal surfaces [10], ultra-thin metal films [11], layered transition-metal chalcogenides [12,13], topological semimetals $[14,15]$ and one-dimensional metals [16], multidimensional materials [17] and graphene [18]. The very high surface sensitivity of HAS for surface dynamic corrugations of the order of $10^{-2}$ a.u. also permits the detection of surface charge-density waves, undetectable by other current surface probes, together with the associated charge density wave (CDW) excitations like phasons and amplitons $[14,19,20]$.

This ability of HAS spectroscopy, including its comparatively high resolution in energy and parallel momentum (see Chap. 9 of Ref. [1]), makes it a choice tool to investigate various aspects of the e-ph interaction in two-dimensional (2D) superconductors. After a theoretical summary (Section 2), with the relevant equations whose detailed derivation is found in refs. $[7,10,11,15,17]$, a few examples for different classes of 2D superconducting materials are discussed in the following sections.

\section{The Electron-Phonon Theory of He-Atom Scattering from Conducting Surfaces}

The repulsive part of the interaction between a He atom and a conducting surface is described to a good approximation by the Esbjerg-Nørskov (EN) potential [21]

$$
V(\mathbf{r})=A n(\mathbf{r}),
$$

where $n(\mathbf{r})$ is the surface electron density, $A=364 \mathrm{eV}_{0}{ }^{3}$ the EN constant, and $a_{0}$ the Bohr's radius [22]. Consider in Figure 1 an inelastic scattering process of a He atom from an initial state $\mid i>$ of energy $E_{i}$ and wave vector $\mathbf{k}_{i}=\left(\mathbf{K}_{i}, k_{i z}\right)$ into a final state $<f \mid$ of energy $E_{f}$ and wave vector $\mathbf{k}_{f}=\left(\mathbf{K}_{f}, k_{f z}\right)$, where $\mathbf{K}_{i}$ and $\mathbf{K}_{f}$ are the components parallel to the surface and $k_{i z}, k_{f z}$ the respective normal components. The inelastic scattering probability $P\left(\mathbf{k}_{i}, \mathbf{k}_{f}\right)$ for one-phonon creation processes in the standard distorted wave Born approximation takes the form (up to a constant factor) [23]:

$$
P\left(\mathbf{k}_{i}, \mathbf{k}_{f}\right) \propto f(\Delta E) N\left(E_{F}\right) \sum_{\mathbf{Q} v} \lambda_{\mathbf{Q} v} \delta\left(\Delta E-\varepsilon_{\mathbf{Q} v}\right),
$$

where $N\left(E_{F}\right)$ is the electron density of states (DOS) at the Fermi level, $\Delta E=E_{f}-E_{i}$ the energy gain, $\varepsilon_{\mathbf{Q} v}$ the phonon energy of wave vector $\mathbf{Q}=\mathbf{K}_{f}-\mathbf{K}_{i}$ and branch index $v$, and $\lambda_{\mathbf{Q} v}$ the mode-selected e-ph coupling constants (mode lambdas) [24,25]. The coefficient

$$
f(\Delta E) \equiv \frac{k_{f}}{\left|k_{i z}\right|} \Delta E\left[1+n_{B E}(\Delta E)\right] A^{2}\left|\left\langle f\left|\psi_{\mathbf{K} n}^{*}(\mathbf{r}) \psi_{\mathbf{K}+\mathbf{Q} n^{\prime}}(\mathbf{r})\right| i\right\rangle\right|^{2},
$$

with $n_{B E}(\Delta E)$ the Bose-Einstein phonon occupation number, is proportional to the square matrix element between final and initial He-atom wave functions of the electron density matrix, connecting two Fermi level electronic states of bands $n$ and $n^{\prime}$ and wave vectors $\mathbf{K}$ and $\mathbf{K}+\mathbf{Q}$, respectively. Please note that the He-atom wave-functions decay very rapidly as they penetrate into the surface electron density near the classical turning point, far away from the first surface atomic plane, where the wave function $\psi_{\mathbf{K} n}(\mathbf{r})$ decays asymptotically as $\mathrm{e}^{-\kappa z}$. In the Wentzel-Kramers-Brillouin (WKB) approxi-mation, $\kappa=\left(2 m^{*} \phi\right)^{1 / 2} \hbar$ where $\phi$ is the surface work function and $m^{*}$ the effective mass of the surface free electrons at the Fermi level. Thus, the square matrix element in Equation (3) can be taken as independent of the electronic band indices and of $\Delta E$, the latter being much smaller than $\phi$. Since at sufficiently high temperature $\Delta E\left[1+n_{B E}(\Delta E)\right] \cong k_{\mathrm{B}} T$, the coefficient $f(\Delta E)$ is also approximately constant, and the inelastic HAS intensity for a phonon $(\mathbf{Q}, v)$ is just proportional to the mode-selected e-ph coupling constant $\lambda_{\mathbf{Q} v}$. This conclusion enables inelastic HAS spectra to indicate which phonons are strongly 
coupled to electrons, and which are less so. As compared to other methods which permit an extraction of the Eliashberg function, the information obtained from HAS spectroscopy is particularly relevant for 2D Bardeen-Cooper-Schrieffer superconductors for providing, besides the frequency, the parallel momentum $\mathbf{Q}$ and branch index $v$ of phonons that are mostly involved in pairing.

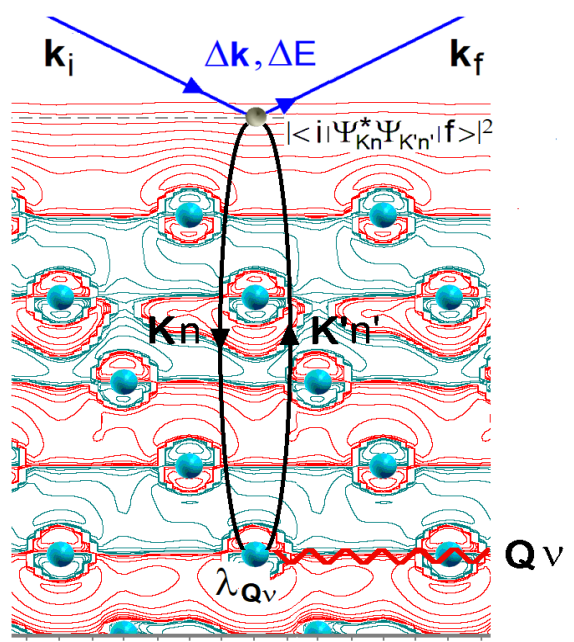

Figure 1. Diagram representing the inelastic scattering of a He atom of incident wavevector $\mathbf{k}_{\mathrm{i}}$ and final wavevector $\mathbf{k}_{\mathrm{f}}$ at the surface of a metal film, where a virtual electron-hole pair is created at the scattering turning point well above the first atomic layer, and recombines into a phonon of wavevector $\mathbf{Q}$ and branch index $v$. The phonon displacement field produces an oscillation of the charge density via the mode-selected e-ph coupling constant $\lambda_{\mathbf{Q} v}$ (red/blue contour lines correspond to positive/negative charge oscillations). The fairly long range of the e-ph interaction determines the depth at which phonons can be created/annihilated by inelastic He-atom scattering (quantum sonar effect). The inelastic amplitude is proportional to $\lambda_{\mathbf{Q} v}$ (mode-lambda spectroscopy) [7].

Another important piece of information is obtained from the temperature dependence of HAS reflectivity. Reflectivity, expressed by the ratio $I(T) / I_{i}$ of the specular peak intensity to the intensity of the incident beam, is attenuated with increasing surface temperature $T$ due to lattice vibrations that modulate the profile of the surface electron density. At conducting surfaces this modulation also contains the contribution of thermal elementary excitations of the electronic Fermi sea, which is however negligible at the usual surface temperatures of HAS experiments. Thus, only phonon excitations, in a first approximation, are responsible for reflectivity attenuation, and this occurs via e-ph interaction. The attenuation of the specular peak intensity is expressed by the Debye-Waller (DW) factor

$$
I(T)=I_{0} e^{-2 W\left(\mathbf{k}_{f}, \mathbf{k}_{i} ; T\right)},
$$

where $I_{0}$ is the rigid surface intensity, and the so-called DW exponent for HAS from insulators, as well as for other probes directly scattered by the atom cores like X-rays and neutrons, is simply related to the mean-square displacement of surface atoms by

$$
2 W\left(\mathbf{k}_{f}, \mathbf{k}_{i} ; T\right)=-\ln \left[I(T) / I_{0}\right]=<\left[\left(\mathbf{k}_{f}-\mathbf{k}_{i}\right) \cdot \mathbf{u}\right]^{2}>_{T}
$$

For a conducting surface the vibrational displacement $\mathbf{u}$ must be replaced by the corresponding modulation of the surface profile at the He-surface classical turning point, proportional to the mean-square phonon displacement. Since the latter includes the contribution of all phonons weighted 
by the respective mode lambdas, it was shown that at sufficiently high temperature, where $\left\langle\mathbf{u}^{2}\right\rangle_{T}$ grows linearly with $T[10,11]$ according to

$$
2 W\left(\mathbf{k}_{f}, \mathbf{k}_{i}, T\right) \cong N\left(E_{F}\right) \frac{\hbar^{2}\left(\Delta k_{z}\right)^{2}}{2 m^{*} \phi} k_{B} T \lambda .
$$

For a two-dimensional electron gas (2DEG) the Fermi level DOS referred to the surface unit cell of area $a_{c}$, is given by $N\left(E_{F}\right)=m^{*} a_{c} / \pi \hbar^{2}$. A multilayer system as considered in the following example is conveniently viewed as a stack of $n_{s}$ 2DEGs, provided $n_{s}$ does not exceed the number $n_{\text {sat }}$ of layers encompassed by the e-ph interaction range. With these definitions, the mass-enhancement factor $\lambda$ (also denoted $\lambda_{\text {HAS }}$ when obtained from HAS DW data) can be written as

$$
\lambda=\frac{\pi}{2 n_{s}} \alpha, \alpha \equiv-\frac{4 \phi}{a_{\mathcal{c}}\left(\Delta k_{z}\right)^{2}} \frac{\partial \ln I(T)}{k_{B} \partial T}, n_{s} \leq n_{\text {sat }} .
$$

\section{Mode-Selected Electron-Phonon Coupling from HAS: Lead Ultra-thin Films}

The early HAS studies of surface phonons in $\mathrm{Pb}(111)$ ultra-thin films (3-8, 10 and 50 monolayers $(\mathrm{ML}))$ grown on a $\mathrm{Cu}(111)$ substrate [26-28] showed something surprising: the number of dispersion curves detected by HAS increases with the number of layers, well beyond the few dispersion curves observed in the semi-infinite crystal. Due to the large acoustic mismatch between $\mathrm{Pb}$ and $\mathrm{Cu}$ (acoustic impedance ratio $Z_{P b} / Z_{C u}=0.56$ ), the observed phonons are confined within the film. Actually the number of modes resolved by HAS reaches a maximum at about $8 \mathrm{ML}$, then it decreases to the four typical surface phonon branches of the face-centered-cubic (111) metal surface localized on the surface atomic bilayer, with prevalent shear-vertical (SV) or longitudinal (L) polarization. This suggests $n_{\text {sat }} \cong 8$ for $\mathrm{Pb}(111)$. Figure $2 \mathrm{a}$, b illustrates the HAS dispersion curves for $5 \mathrm{ML}$ - and $7 \mathrm{ML}-\mathrm{Pb}(111) / \mathrm{Cu}(111)$ (filled circles) as compared to the theoretical dispersion curves calculated with DFPT calculations [7]: substantially all branches with prevalent SV polarization are detected. The calculated eigenvectors indicate that the highest mode $\left(\varepsilon_{2}\right)$ is localized on the surface bilayer, whose interlayer distance is quite contracted, whereas the second highest mode $\left(\varepsilon_{1}\right)$ is localized near the film-substrate interface, a few layers beneath the surface. This offers a clear demonstration of the quantum sonar effect.

The inelastic HAS spectra measured in the $90^{\circ}$ planar scattering geometry along the scan curves at incident angles of $37^{\circ}$ and $39.5^{\circ}$ for $5 \mathrm{ML}$ (Figure 2a), and $35.5^{\circ}$ and $37.5^{\circ}$ for $7 \mathrm{ML}$ (Figure $2 \mathrm{~b}$ ) are displayed in Figure 2c,e, respectively. The spectra of the mode-lambdas $\lambda_{\mathbf{Q} v}$ calculated with DFPT for all the theoretical quasi-SV modes intersected by the scan curves (Figure $2 \mathrm{~d}, \mathrm{f}$ ) compare very well with the experimental inelastic spectra, thus demonstrating the approximate proportionality predicted by Equation (2).

The calculated $\lambda_{\mathbf{Q} v}$ for the different modes $\left(v=\alpha_{1}, \alpha_{2}, \ldots, \varepsilon_{1}, \varepsilon_{2}\right)$ are plotted as functions of $\mathbf{Q}$ in Figure 3 for both $5 \mathrm{ML}$ and $7 \mathrm{ML}$. It is rather instructive that the maxima of $\lambda_{\mathbf{Q} v}$ occur for the $\mathbf{Q}=0$ acoustic modes $\alpha_{1}$. These modes have a small but finite frequency at $\mathbf{Q}=0$ (Figure $2 \mathrm{a}, \mathrm{b}$ ), because of the film-substrate interaction, and consist of an almost rigid translation of the whole film against the substrate. Since the wave penetration into the substrate is quite small, due to the large acoustic mismatch, the displacement field gradient, measuring the local stress, is concentrated at the interface, and therefore a large contribution to the e-ph coupling comes in this case from the interface electrons. 

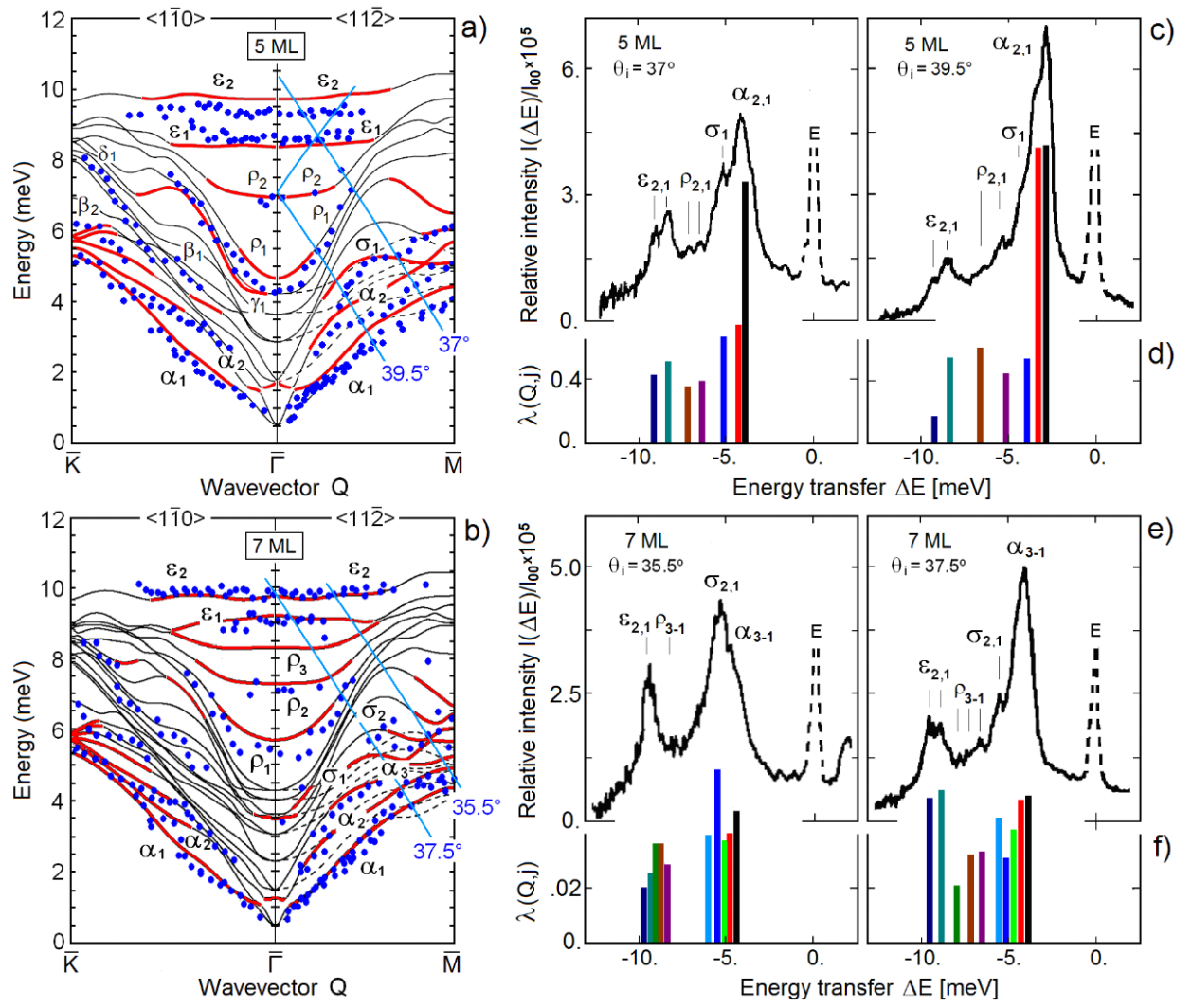

Figure 2. The experimental HAS phonon dispersion curves (•) for (a) $5 \mathrm{ML}$ and (b) $7 \mathrm{ML}$ of Pb grown on a $\mathrm{Cu}(111)$ substrate compared with the theoretical DFPT dispersion curves. The heavier (red) lines refer to quasi-shear-vertical modes, thin lines are for quasi-longitudinal modes, broken lines for shear-horizontal modes, not observable in the planar HAS configuration. The intersections of the two HAS scan curves with the dispersion curves for incident angles of $37^{\circ}$ and $39.5^{\circ}$ (a) and of $35.5^{\circ}$ and $37.5^{\circ}(\mathbf{b})$ provide the energy and momentum of the observed phonons. The corresponding HAS energy-gain spectra $(\mathbf{c}, \mathbf{e})$ show some prominent peaks, approximately proportional to the respective mode lambdas $\lambda_{\mathbf{Q} v}$ calculated with DFPT (d,f); the colors correspond to those of the $\lambda_{\mathbf{Q} v}$ plots in Figure 3. E labels the diffuse elastic peaks (broken lines). a,b reproduced with permission from [7], copyright 2011 by the American Physical Society. c-f from [9], with permission from The Royal Society of Chemistry.
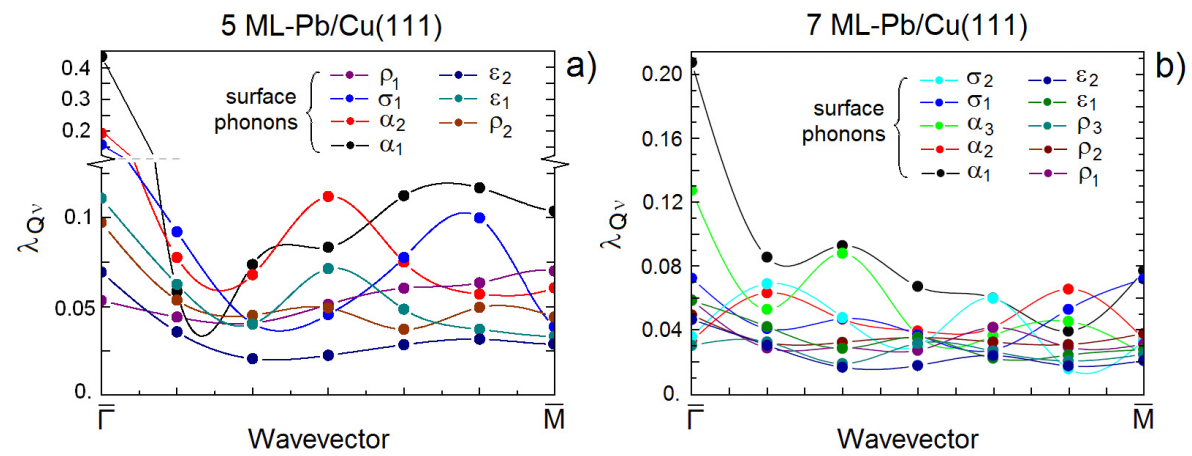

Figure 3. Plots of the mode-selected e-ph coupling constants $\lambda_{\mathbf{Q} v}$ for different branch indices $v$ as functions of the wavevectors $\mathbf{Q}$ in the $\overline{\Gamma M}$ direction for $5 \mathrm{ML}-\mathrm{Pb} / \mathrm{Cu}(111)$ (a) and $7 \mathrm{ML}-\mathrm{Pb} / \mathrm{Cu}(111)(\mathbf{b})$, calculated with DFPT [7] (reproduced with permission, copyright 2011 by the American Physical Society). Indexed Greek letters label the same modes of Figure 2a,b; colors correspond to those of the $\lambda_{\mathbf{Q} v}$ bins in Figure 2d,f. 
Measurements of the temperature-dependent DW exponent during the layer-by-layer deposition of $\mathrm{Pb}$ films on $\mathrm{Cu}(111)$ permit the obtaining of $\lambda$ as a function of the number of layers via Equation (7) (Figure 4a) [27]. Please note that due to oscillating surface relaxation where the interlayer distance oscillates so as to form a sequence of atomic bilayers near the surface, the reflectivity peaks actually occur at $n=1$ (wetting layer), 3, 4.5, 6.5, $8 \ldots$ ML. As appears in Figure $4 \mathrm{a}, \lambda<0.8$ for a single $\mathrm{Pb}$ wetting layer and for $3 \mathrm{ML}$, then rises to values above 1 for $n \geq 4 \mathrm{ML}$, exhibiting quantum-size oscillations [26]. This behavior corresponds quite well to what has been reported from scanning-tunneling microscopy/spectroscopy (STM/STS) for the superconducting $T_{c}$ of $\mathrm{Pb}$ ultra-thin films grown on semiconductor surfaces (Figure 4b) [29-34].
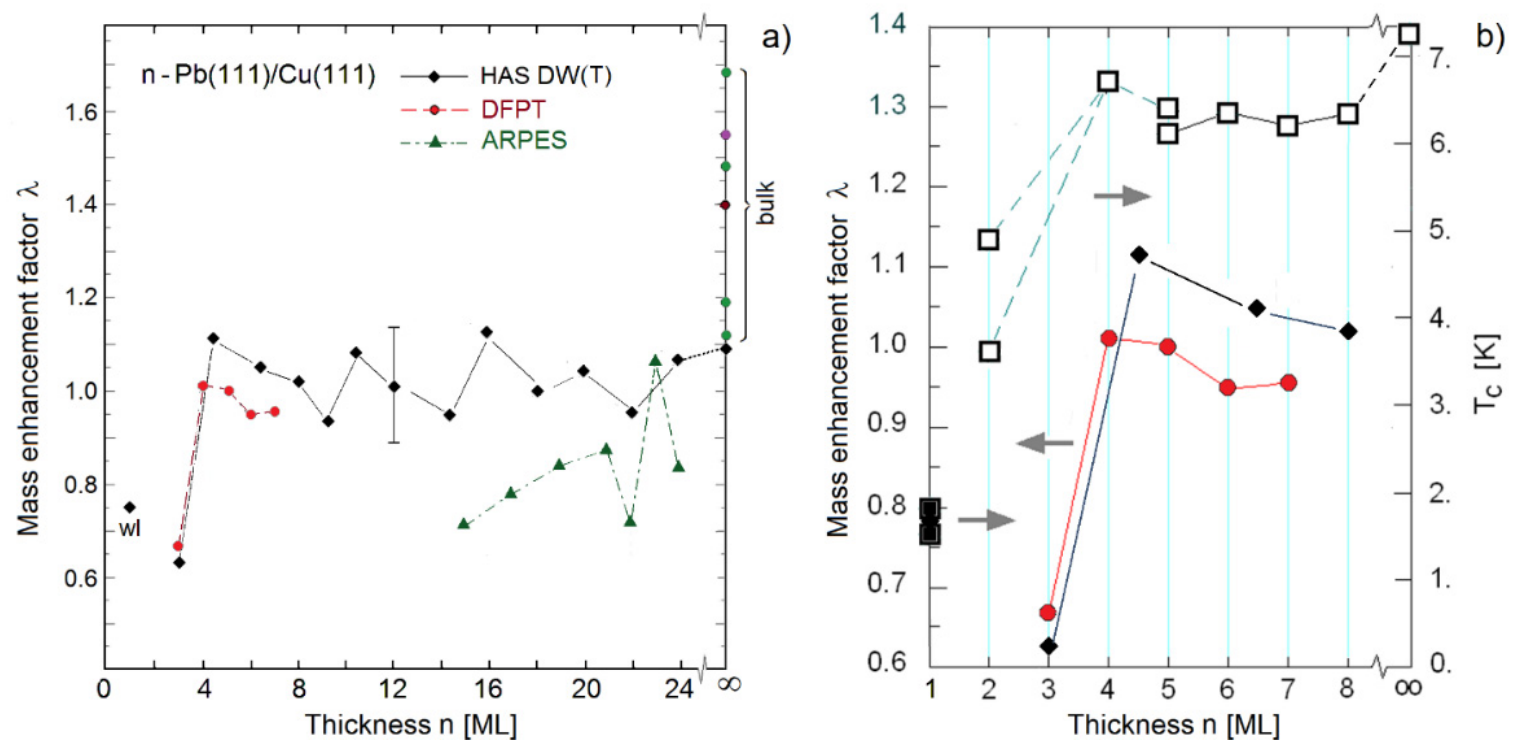

Figure 4. (a) Values of the mass-enhancement factor $\lambda$ extracted from the temperature dependence of the HAS Debye-Waller exponent, measured for a He-beam incident energy of $5.97 \mathrm{meV}(\bullet)[11,26,27]$, (reproduced with permission, copyright 2014 by the American Chemical Society) are compared to values from ARPES measurements $(\boldsymbol{\Lambda})$ [35] and from density functional perturbation theory $(\bullet)$ [7]. The HAS value at $n=1 \mathrm{ML}$ refers to the $\mathrm{Pb}$ wetting layer (wl). A collection of values for bulk $\mathrm{Pb}$ taken from literature [24,25] (filled circles at $n=\infty$ ) are shown for comparison. (b) The experimental HAS $(\bullet$ ) and theoretical $(\bullet$ ) values of $\lambda$ shown in (a) for $n \leq 8 \mathrm{ML}$ are compared with the experimental superconducting $T_{c}$ of ultra-thin $\mathrm{Pb}$ films on $\mathrm{Si}(111)$ [9] (with permission from The Royal Society of Chemistry) for two different equilibrium configurations of the $2 \mathrm{ML}$ and for $n=4,5 \mathrm{ML}$ (open squares) [31] and of the wetting layer, $n=1 \mathrm{ML}$ (filled squares) [33].

For 3 to $8 \mathrm{ML}$ (Figure $4 \mathrm{~b}$ ) the HAS data reproduce quite well the results of DFPT calculations [7]. For thicknesses of 15 to $24 \mathrm{ML}$, the HAS data in Figure 4 a show quantum-size oscillations that look different from those of the corresponding angle-resolved photoemission spectroscopy (ARPES) data [35]. It should be noted that ARPES values for $\lambda$ are derived from the deformation of an electronic band induced by the e-ph coupling near the Fermi surface, and therefore refer to that specific electronic band, whereas the HAS values from the DW exponent are averages over all possible virtual phonon-induced electronic transitions around the Fermi level, as considered in the definition of $\lambda$.

\section{A Quasi-1D Metal: Bi(114)}

Although bulk crystalline bismuth at normal pressure is not superconducting above $0.53 \mathrm{mK}[36,37]$, nanostructured [38] and amorphous bismuth [37] are reported to become superconductors with critical temperatures above $1 \mathrm{~K}$. It is, therefore, interesting to investigate the e-ph interaction in Bi nanostructures by means of HAS. Of particular interest is the (114) surface of bismuth (Figure 5a). 
Bi(114) exhibits, in its stable reconstructed $(1 \times 2)$ form, resulting from a missing-row transition, the features of a one-dimensional (1D) topological metal consisting of conducting atomic rows $28.4 \AA$ apart $[15,38]$. Below a critical temperature of $\approx 240 \mathrm{~K}$, a CDW arises along the rows with a critical exponent of $1 / 3$ due to a Peierls dimerization along the chains [16], leading to a $(2 \times 2)$ reconstruction with an oblique unit cell in real (Figure 5a) and reciprocal space (Figure 5b). The electronic structure in the $(1 \times 2)$ phase, calculated by Wells et al. [39], is characterized at the Fermi level by carrier pockets at the zone center $\bar{\Gamma}$ and the zone-boundary points $\bar{X}$ (Figure $5 \mathrm{c}$ ). The $\bar{\Gamma}-\bar{X}$ intervalley e-ph coupling explains the $(2 \times 2)$ reconstruction.

a)

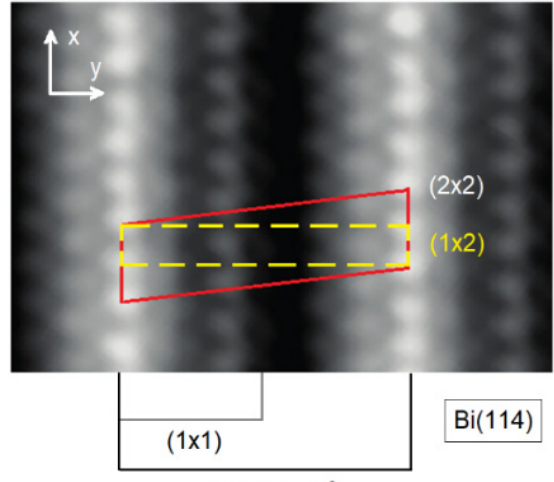

b)

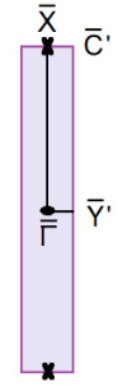

(1x2) $T>T_{c}$

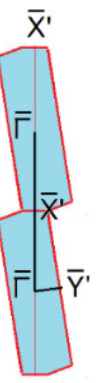

(2x2) $T<T_{c}$

$(1 \times 2) 28.4 \AA$

C)
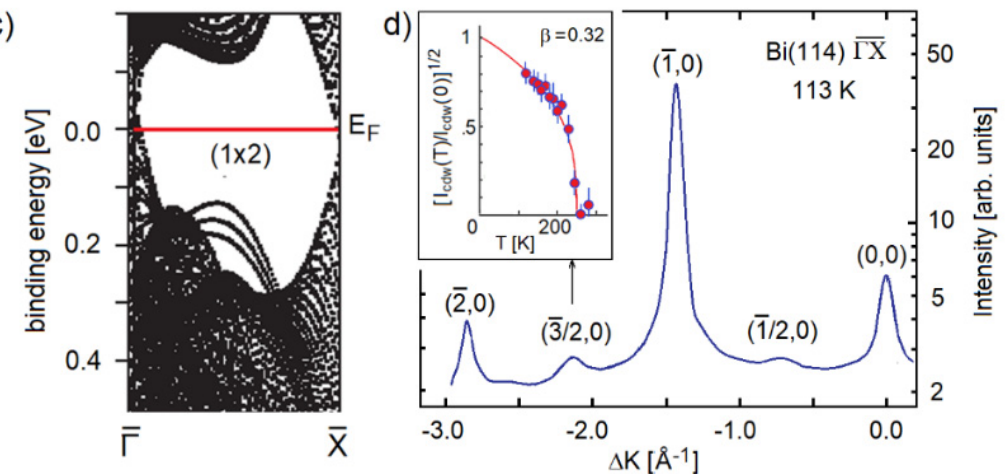

Figure 5. (a) STM image of the $(1 \times 2) \mathrm{Bi}(114)$ surface resulting from a missing-row reconstruction, leading to parallel atomic rows in the $x$-direction, $28.4 \AA$ apart, that confers a quasi-1D metallic character to the surface [39]. Below $\approx 140 \mathrm{~K}$ the atomic rows undergo a Peierls dimerization with an oblique $(2 \times 2)$ unit cell in real (a) and reciprocal space (b). Dimerization is due to intervalley nesting between Fermi level pockets at $\bar{\Gamma}$ and $\bar{X}$ points of the $(2 \times 1)$ Brillouin zone (c). The transition is monitored by the intensity of HAS diffraction from semi-integer CDW G-vectors (d) as a function of temperature. The inset of panel (d) shows that the order parameter grows with a critical exponent $\beta \cong 1 / 3$ [15] published 2020 by the American Chemical Society).

The HAS diffraction spectra of $\mathrm{Bi}(114)$ along the row direction, reported in $[15,16]$, show the temperature evolution of the CDW peaks, the most prominent being that at $( \pm 3 / 2,0)$; an example, measured at $113 \mathrm{~K}$, is shown in Figure $5 \mathrm{~d}$. The best fit of the order parameter, proportional to $\left[I_{\mathrm{CDW}}(T) / I_{\mathrm{CDW}}(0)\right]^{1 / 2}$ for the CDW peak (inset of Figure $5 \mathrm{~d}$ ), yields a CDW critical temperature of $242 \pm 7 \mathrm{~K}$ and a critical exponent $\beta=0.32 \pm 0.03$, consistent with the universal exponent of $1 / 3$ as predicted in the presence of fluctuations $[40,41]$, and in agreement with the critical exponent for other CDWs in layered chalcogenides and quasi-1D systems [17,42-45].

The e-ph coupling constant $\lambda$ can be derived from the temperature dependence of the DW exponent, Equation (7), with the experimental parameters taken from Ref. [16]: $a_{c}=129 \AA^{2},\left(\Delta k_{z}\right)^{2}=54.24 \AA^{-2}$, $\phi=4.23 \mathrm{eV}$ (for $\mathrm{Bi}$ ), $-\partial \ln I(T) / k_{\mathrm{B}} \partial \mathrm{T}=104.3 \mathrm{eV}^{-1}$ and $n_{\mathrm{S}}=1$ (assuming that only the surface atomic rows 
contribute). This gives $\lambda=0.40$, to be compared with the HAS value 0.57 for Bi(111) [10]. A slightly larger value for $\mathrm{Bi}(114), \lambda=0.45$, is obtained when treated as a $1 \mathrm{D}$ free-electron gas system, as discussed in Ref. [17]. This value is probably more reliable, in consideration of the quasi-1D metallic nature of $\mathrm{Bi}(114)(1 \times 2)$, although the difference is well within the uncertainty of the present method.

\section{Superconducting Layered Chalcogenides}

The large family of layered chalcogenides includes several superconductors. Although their superconductivity generally occurs at fairly low temperatures and under special conditions (proximity, pressure, high doping, low dimensionality), their great interest is related to the frequent coexistence/competition with CDWs and the interplay of electron-electron and e-ph interactions.

The high sensitivity and resolution of HAS permit detection of CDWs where other conventional surface probes fail, and measurement of the dispersion curves of their collective excitations $[14,16,19]$. The additional possibility to directly estimate the e-ph interaction makes HAS an excellent tool to investigate superconducting (SC) layered chalcogenides.

\section{1. $1 T-\mathrm{TaS}_{2}(001)$}

Tantalum di-chalcogenides as superconductors exemplify the special conditions mentioned above. The SC critical temperature of $1 \mathrm{~T}-\mathrm{TaS}_{2}(001)$, originally reported by Wilson et al. to be about $0.5 \mathrm{~K}$ [46], was later determined to be about $1.5 \mathrm{~K}$ at normal pressure [47]. Pressure was shown by Sipos et al. [48] to produce a large increase of $T_{c}$ up to $5 \mathrm{~K}$ at $5 \mathrm{GPa}$ and then to keep it about constant at larger values up to $25 \mathrm{GPa}$. A raise of $T_{c}$ can also be obtained, as recently demonstrated [49,50], by reducing the sample thickness to a few layers, and even in a monolayer an increase of $T_{c}$ up to $3.61 \mathrm{~K}$ is induced by the formation of structural defects [51]. An alternate stacking of the $1 \mathrm{~T}$ and $2 \mathrm{H}$ layers of $\mathrm{TaS}_{2}$ to form the so-called $4 \mathrm{Hb}$ polytype was also shown to raise $T_{c}$ to $2.7 \mathrm{~K}$ [52]. The coexistence of the $\mathrm{CDW}$ with superconductivity in $1 \mathrm{~T}-\mathrm{TaS}_{2}$ has been investigated by Sipos et al. [48] and more recently by Ritschel et al. [53]. Wagner et al. [54] reported on the interesting observation that a $\mathrm{Cu}$ doping of $2 \mathrm{H}-\mathrm{TaS}_{2}$ raises the $\mathrm{SC} T_{c}$ from $0.5 \mathrm{~K}$ of the undoped material to a maximum of $4.5 \mathrm{~K}$ for a $4 \%$ doping, while dramatically reducing the CDW coherence length by a factor ten.

The complex CDW structure of 1T-TaS 2 , as described by Wilson et al. [46,55], Coleman et al. [56], and more recently by $\mathrm{Yu}$ et al. [57], has been studied with HAS by Cantini et al. [58] and by Heimlich et al. $[59,60]$. The slopes of the HAS specular intensity as a function of temperature, shown in Figure 6a [59] for increasing temperature in the low- $T$ commensurate CDW (C-CDW), intermediate-T nearly commensurate CDW (NC-CDW) and high- $T$ incommensurate CDW (I-CDW) regions, permit to obtain from Equation 7 the respective e-ph coupling constant. With the input data $k_{i z}{ }^{2}=14.58 \AA^{-2}$ [60], $\phi=5.2 \mathrm{eV}$ [61], $a_{c}=9.43 \AA^{2}$ [62] and $n_{s}=2$, from a Thomas-Fermi screening length not exceeding $1 \mathrm{~nm}[57]$, the observed specular intensity slopes give $\lambda(C-C D W)=0.61 \pm 0.06, \lambda(\mathrm{NC}-\mathrm{CDW})=0.91 \pm 0.09$, and $\lambda(\mathrm{I}-\mathrm{CDW})=0.61 \pm 0.10$ for the three phases. The data for decreasing temperature, presented in [52], give for the I-CDW and NC-CDW comparable values within the experimental error. For the low temperature C-CDW phase, the McMillan formula yields, with $\mu^{*}=0.20$ [63] and a Debye temperature $\Theta_{\mathrm{D}}$ of $249 \mathrm{~K}[46,64,65]$, a superconducting critical temperature $T_{\mathcal{c}}=1.2 \mathrm{~K}$, in fair agreement with the value at normal pressure of $1.5 \mathrm{~K}$ reported by Nagata et al. [47]. 

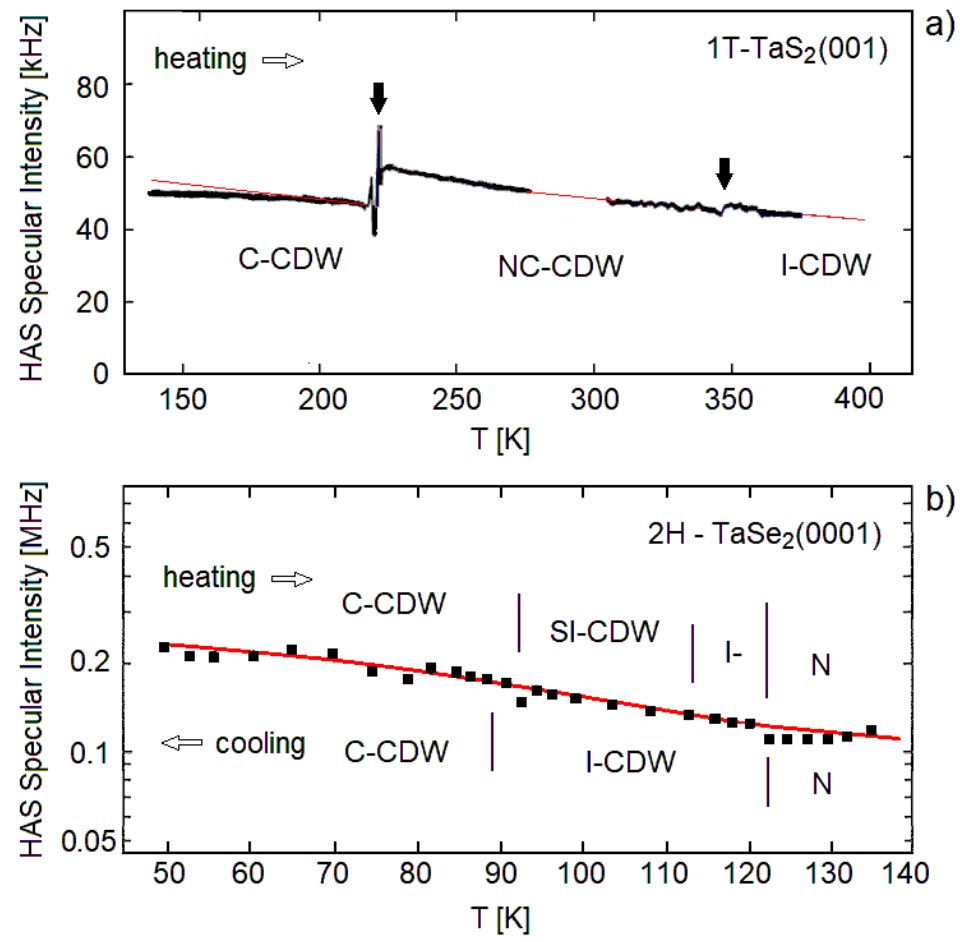

Figure 6. Thermal attenuation (Debye-Waller factor) data for scattering of He atoms from two chalcogenide surfaces. The specular intensity is plotted as a function of temperature. (a) $1 \mathrm{~T}-\mathrm{TaS}_{2}(001)$ plotted on a linear scale, and (b) $2 \mathrm{H}-\mathrm{TaSe}_{2}(0001)$ plotted on a logarithmic scale. In (a), the crystal was initially at low temperature and then heated, and the two vertical arrows indicate the charge density wave transitions. The transition from incommensurate charge density wave (I-CDW) to non-commensurate (NC-CDW) occurs at about $350 \mathrm{~K}$, while the transition from the NC-CDW to the commensurate (C-CDW) phase occurs at about $220 \mathrm{~K}$. The three red segments indicate the slopes in the C-CDW phase near the transition, and the average slopes in the NC-CDW and I-CDW phases. In (b) the experimental data are also shown for increasing temperature from the C-CDW through the transitions to the striped incommensurate (SI-CDW) at $92 \mathrm{~K}$, the I-CDW at $113 \mathrm{~K}$, and finally the normal phase at $122 \mathrm{~K}$, as marked by the vertical lines. Also marked are the transition temperatures for decreasing $T$, with relevant hysteresis effects: no SI-CDW is observed and the I-CDW to C-CDW occurs at $88 \mathrm{~K}$ [59] (adapted from [17]). The red line is a smooth interpolation of the data points.

For the high-pressure (5 GPa) NC-CDW phase, Rossnagel derives from the McMillan formula and the input data $T_{c}=5 \mathrm{~K}, \mu^{*}=0.20$ and $\Theta_{\mathrm{D}}=249 \mathrm{~K}$, an e-ph interaction constant $\lambda=0.85$ [63], also in agreement with the present value of $\lambda$ for the NC-CDW phase, although HAS experiments are performed in vacuum conditions. Density functional theory calculations by Amy Liu [66] also predict a dramatic effect of pressure, with $\lambda$ varying from 2.09 at $5 \mathrm{GPa}\left(T_{\mathcal{c}}=16 \mathrm{~K}\right)$ to 0.69 at $30 \mathrm{GPa}\left(T_{\mathcal{c}}=5.9 \mathrm{~K}\right)$. Please note that the Debye temperature, here corresponding to a phonon energy of $21.6 \mathrm{meV}$, is adopted in the McMillan formula as an average energy of phonons contributing to $\lambda$, and may have little to do with that derived from the specific heat, according to Debye theory. Moreover, this theory has been conceived for monoatomic crystals with only three acoustic phonon branches, whereas for the additional flat optical branches of polyatomic crystals the Einstein model would be more appropriate. The simple fit of the Debye theory to polyatomic crystals with high-energy optical phonon branches leads to an important temperature dependence of $\Theta_{\mathrm{D}}$. Since in general $T_{c}<<\Theta_{\mathrm{D}}$, it is implicit that $\Theta_{\mathrm{D}}$ should be conveniently taken in its low- $T$ limit. In the present three-atomic case, the Debye phonon energy $\varepsilon_{\mathrm{D}}=k_{\mathrm{B}} \Theta_{\mathrm{D}}=21.6 \mathrm{meV}$ falls just above the acoustic region but well below the optical phonon region (27-40 meV), according to pseudo-charge model calculations for the C-CDW phase in its $(\sqrt{ } 13 \times \sqrt{ } 13) R 13.9^{\circ}$ superstructure $\left(1 \mathrm{~T}-\mathrm{TaS}_{2}\right)$ [67]. This is indicative of a relevant contribution of 
acoustic phonons to the e-ph interaction, consistently with the fact that HAS measurements of the surface phonon dispersion curves of $1 \mathrm{~T}-\mathrm{TaS}_{2}$ show the Rayleigh wave branch with several umklapp replicas corresponding to CDW reciprocal lattice vectors [68].

\section{2. $2 \mathrm{H}-\mathrm{TaSe}_{2}(0001)$}

HAS reflectivity data for $2 \mathrm{H}-\mathrm{TaSe}_{2}$ (Figure $6 \mathrm{~b}$ ) show a decrease for increasing temperature similar to that of $1 \mathrm{~T}-\mathrm{TaS}_{2}$, with imperceptible discontinuities at the CDW transition temperatures. The HAS intensities of the CDW diffraction peaks $[59,60]$ show instead clear features at the transition temperatures, from the C-CDW to the striped-incommensurate (SI-CDW) phase (92 K), from the SI-CDW to the I-CDW phase (113 K) and from the I-CDW to the normal phase $(122 \mathrm{~K})$, in agreement with previous McWhan et al. X-ray data [69]. Similar agreement is found for the transition temperatures during cooling, with hysteresis at the I-CDW $\rightarrow$ C-CDW transition $(88 \mathrm{~K})$. The decay slopes of the specular intensity for increasing temperature (Figure $6 \mathrm{~b}$ ) in the C-CDW linear region (70-90 K) and in the (SI + I) - CDW region (90-122 K) are about the same, within the experimental uncertainty. With the input data $k_{i z}{ }^{2}=68.45 \AA^{-2}[59,60], \phi=5.5 \mathrm{eV}$ [70], $a_{c}=10.22 \AA^{2}$ [71] and $n_{s}=2$ (from a spherically averaged coherence length of $\approx 14 \AA$ [72], vs. the unit cell thickness including two Ta layers of $12.70 \AA$ ), a single value for $\lambda=0.57 \pm 0.08$ is obtained. The data for decreasing temperature displayed in [59] show a smaller slope in the I-CDW region (122-88 K), giving $\lambda=0.30 \pm 0.06$, but approximately the same slope in the $\mathrm{C}-\mathrm{CDW}$ region $(<88 \mathrm{~K})$. The factor of two difference in $\lambda$ found in the incommensurate phase between heating and cooling data may reflect the structural dependence on the thermal history, as suggested by the fact that the SI phase is observed when heating and not when cooling the sample. In any case the superconducting temperature resulting from McMillan's formula, with a Debye temperature of $140 \mathrm{~K}$ [73], $\mu^{*}=0.20$, and $\lambda=0.57$ for the C-CDW phase is equal to $410 \mathrm{mK}$. The C-CDW values of $\lambda$ and the corresponding $T_{\mathcal{c}}$ are larger than those found in the literature, e.g., $\lambda=0.49$ reported by Rossnagel [64], 0.39 by Bhoi et al. [73], 0.4 by Luo et al. [74], and correspondingly rather small values of $T_{c}$, such as $200 \mathrm{mK}$ by Kumakura et al. [75] and $130 \mathrm{mK}$ by Yokota et al. [76].

As in the case of $1 \mathrm{~T}-\mathrm{TaS}_{2}$ the interplay between CDW and superconductivity is an interesting issue, as discussed in recent works by Lian et al. [77], Wu et al. [78], Liu et al. (for 1T- $\mathrm{TaSe}_{2-\mathrm{x}} \mathrm{Te}_{\mathrm{x}}$ ) [79], etc. Similarly, a large rise of $T_{c}$ up to above $2 \mathrm{~K}$ has been demonstrated as an effect of doping [80] or ion gating (intercalation) [78], as well as of film thickness reduction down to $1 \mathrm{ML}$. For example, Lian et al. have shown that a value of $\lambda=0.4$, valid for bulk $2 \mathrm{H}-\mathrm{TaSe}_{2}$ and giving a $T_{c}$ of $100 \mathrm{mK}$, rises to $\lambda=0.74$ in a single monolayer, with a $T_{c}=2.2 \mathrm{~K}$ [78]. Thus, the larger values of $\lambda$ observed with HAS clearly refer to the surface, more precisely to the surface bilayer $\left(n_{s}=2\right)$.

The conjecture, originally formulated for graphite, that in layered crystals with weak interlayer van der Waals forces the surface phonon dispersion curves should be almost coincident with those of the bulk, proved invalid for layered chalcogenides (Figure 7) [1]. A specific role of e-ph interaction at the surface of $2 \mathrm{H}-\mathrm{TaSe}_{2}$, and in general of transition-metal chalcogenides, has been surmised since the early HAS measurements of the surface phonon dispersion curves $[43,81,82]$. An intriguing HAS observation in $2 \mathrm{H}-\mathrm{TaSe}_{2}$ was a temperature-dependent Kohn anomaly in the Rayleigh wave ( $\left.\mathrm{RW} \equiv \mathrm{S}_{1}\right)$ dispersion curve at about $\frac{1}{2} \overline{\Gamma M}$ [82], whereas the bulk longitudinal acoustic $\left(\Sigma_{1}\right)$ mode, as measured with inelastic neutron spectroscopy [70], shows a T-dependent anomaly at ${ }^{2} / 3 \overline{\Gamma \mathrm{M}}$ (Figure 7a). Moreover, the maximum softening observed in the bulk $\Sigma_{1}$ mode occurs at the normal-to-CDW transition $(\approx 120 \mathrm{~K})$, whereas the deepest $S_{1}$ Kohn anomaly occurs at $\approx 110 \mathrm{~K}$. Actually the HAS intensities of the CDW diffraction peaks [43] exhibit hysteresis loops around the I $\leftrightarrow$ SI transition temperature (113 K), which would associate in some way the SI phase to the surface region, within the penetration depth of the RW at $\frac{1}{2} \overline{\Gamma M}$. As mentioned above, HAS data on the temperature dependence of the CDW diffraction peaks also provided the order-parameter critical exponent for the CDW transition at $122 \mathrm{~K}$ equal to the universal $\beta=1 / 3$ [42], as generally found for CDW transitions driven by e-ph interaction in this class of materials. 


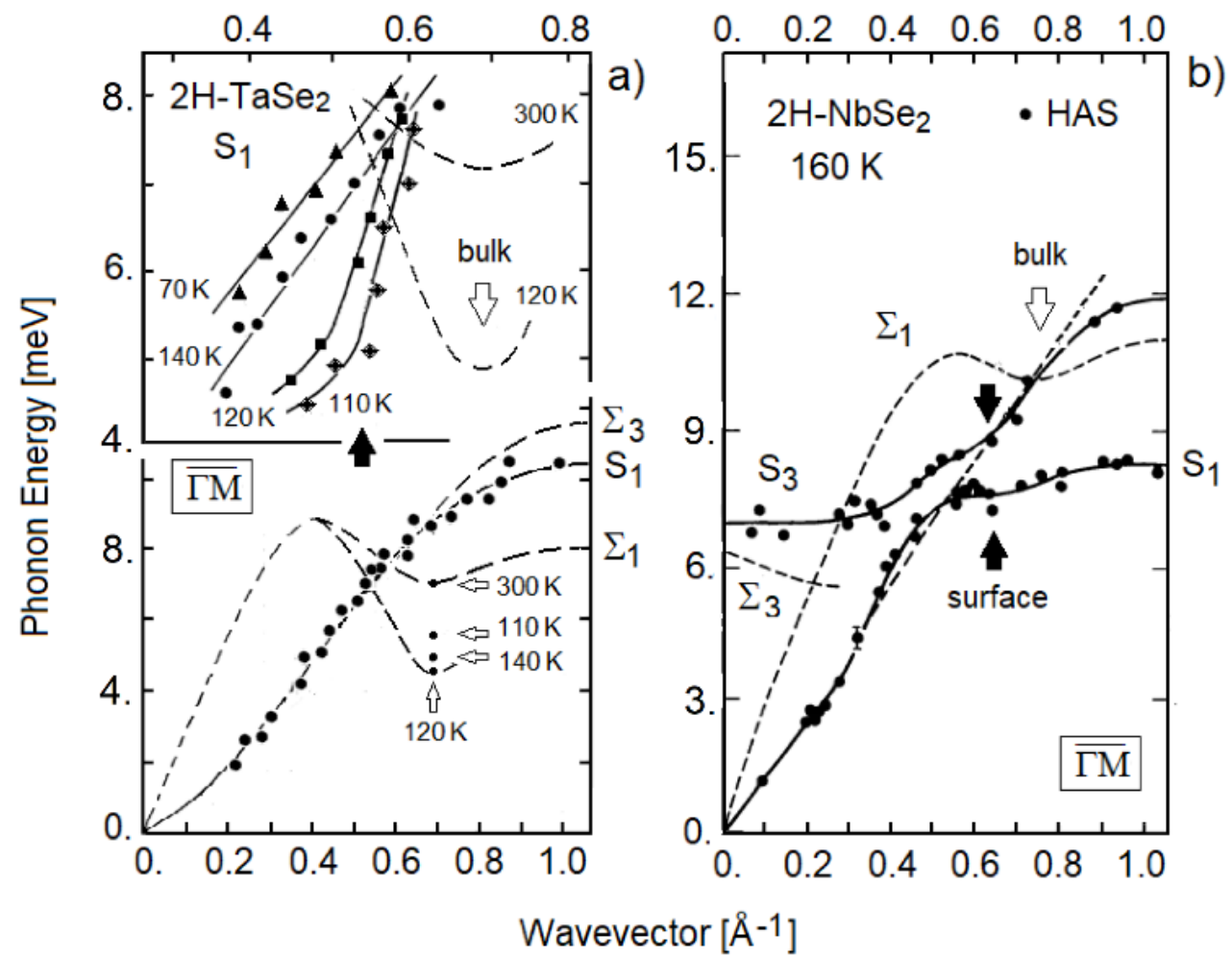

Figure 7. (a) The Rayleigh wave dispersion curve $\left(\mathrm{S}_{1}\right)$ in $2 \mathrm{H}-\mathrm{TaSe}_{2}$ measured with HAS (larger dots) $[81,82]$ at $140 \mathrm{~K}$ along the $\overline{\Gamma M}$ direction (bottom) and at different temperatures around $\frac{1}{2} \overline{\Gamma M}$ (top), where a Kohn anomaly develops with a minimum at $110 \mathrm{~K}$ (upward filled arrow), close to the I-CDW $\rightarrow$ SI-CDW transition. For comparison the deep anomaly in the bulk longitudinal acoustic mode $\left(\Sigma_{1}\right)$ (broken lines) is located at $2 / 3 \overline{\Gamma \mathrm{M}}$ and reaches its minimum at about the I-CDW $\rightarrow \mathrm{N}$ transition temperature of $\sim 120 \mathrm{~K}$ (open downward arrow) [71]. (b) A similar situation is found in HAS data for $2 \mathrm{H}-\mathrm{NbSe}_{2}$ measured at $160 \mathrm{~K}$ [83], where the shift from the bulk anomaly at about $3 / 4 \overline{\Gamma \mathrm{M}}$ [71] (open downward arrow) to the surface anomalies at $2 / 3 \overline{\Gamma M}$ in both the Rayleigh wave $\left(\mathrm{S}_{1}\right)$ and the optical $S_{3}$ branches (filled black arrows) is smaller than in $2 \mathrm{H}-\mathrm{TaSe}_{2}$, although the anomalous $\mathrm{S}_{1}$ mode softens at lower temperature down to zero $[84,85]$.

The shift of the surface phonon anomaly to a wave vector different from that for the bulk phonon anomaly may be ascribed to a different nesting of the Fermi level surface electronic states with respect to that for the bulk states. A similar behavior is found with $\mathrm{HAS}$ in $2 \mathrm{H}-\mathrm{NbSe}_{2}$ (Figure $7 \mathrm{~b}$ ), where the dip in the dispersion curve of the bulk $\Sigma_{1}$ phonon also falls at $\approx 0.76 \AA^{-1}$ while the dips of both $S_{1}$ and $\mathrm{S}_{3}$ surface phonons branches, as measured by HAS, are shifted to a smaller wave vector, $\approx 0.65 \AA^{-1}$. It is unfortunate that this HAS data, originally measured in 1986 by Brusdeylins and Toennies and reproduced in [83], have not been extended to lower temperatures, where, as found with inelastic X-ray spectroscopy (IXS) $[84,85]$, the $S_{1}$ frequency at $\approx 0.65 \AA^{-1}$ vanishes at about $33 \mathrm{~K}$. The Weber et al. analysis based on state-of-the-art ab-initio calculations indicate the wave vector dependence of the e-ph coupling as the driving mechanism for CDW formation in $2 \mathrm{H}-\mathrm{NbSe}_{2}$ [85]. Considering the superior resolution of inelastic HAS spectroscopy in the meV range with respect to IXS, and its ability to obtain direct information on e-ph coupling constants, revisiting with HAS the CDW phases and transitions in this class of 2D superconductors would be highly desirable. Recent examples in this direction are the HAS measurements of $\lambda$ in $2 \mathrm{H}-\mathrm{MoS}_{2}$ [12], 1T-PtTe 2 [13] and 1T-PdTe 2 [86].

\subsection{Pnictogen Chalcogenides}

Layered pnictogen chalcogenides, with a bulk electronic band gap and a cone of topological Dirac states localized at the surface and crossing the Fermi level, are topological insulators (TI) unsuitable 
to be superconductors at any temperature. They can however become so by intercalating donor atoms [87-89], doping [90], by interfacing with conventional superconductors via the proximity effect [91-96], or under pressure [97-99]. Although critical temperatures of induced superconductivity are in the best cases in the range of a few Kelvin, the involvement of surface topologically protected states confers a particular stability against external disturbances. This would make pnictogen chalcogenides and their heterostructures particularly suitable for device applications. Moreover, TI-superconductor heterostructures have provided a quantum playground for some fundamental advances, e.g., the evidence of Majorana fermions in condensed matter with promising applications to quantum computing (for brief accounts see, e.g., [100-102]).

A successful option was looking for Majorana fermions in the vortices of a 2D chiral topological superconductor [103-109]. Xu et al. [110], were able to detect with STM/STS a Majorana mode in a heterostructure made of the topological insulator $\mathrm{Bi}_{2} \mathrm{Te}_{3}$ and the superconductor $\mathrm{NbSe}_{2}$. The spin-selected Andreev reflection effect [111] was exploited by Sun et al. [112] in a similar 2D heterostructure where five quintuple layers of the topological insulator $\mathrm{Bi}_{2} \mathrm{Se}_{3}$ are interfaced to a $\mathrm{NbSe}_{2}$ superconductor, which enabled unveiling Majorana zero modes by probing the vortex core states with spin-polarized-STM/STS. An important issue is the coexistence of superconductivity with the topological order [113-115]. The recent HAS observation of diffraction features attributed to long-period CDWs in $\mathrm{Bi}_{2} \mathrm{Se}_{3}$ [19] raises another coexistence issue, as mentioned above for layered transition-metal chalcogenides, with the corresponding question about the origin and role of e-ph interaction in topological pnictogen chalcogenides.

HAS reflectivity measurements on $\mathrm{Bi}_{2} \mathrm{Se}_{3}(111), \mathrm{Bi}_{2} \mathrm{Te}_{3}(111), \mathrm{Bi}_{2} \mathrm{Te}_{2} \mathrm{Se}(111)[15,116,117]$ and $\mathrm{Sb}_{2} \mathrm{Te}_{3}(111)$ [118] have permitted obtaining $\lambda$ from the temperature dependence of the DW exponent and Equation (7) $\left(\lambda_{\text {HAS }}\right.$ in Figure 8b). Their respective values are 0.23, 0.19, 0.14 and 0.08, all with an experimental uncertainty of around $10 \%$. The large range of values as compared to the similarity of these compounds can be understood by considering their electronic structure at the Fermi level, as resulting from the ARPES data for the bismuth chalcogenides used in HAS experiments $[15,19,116]$ and from ab-initio calculations for $\mathrm{Sb}_{2} \mathrm{Te}_{3}(111)$ [118] (Figure 8a,c). The substantial differences in the sets of topological states concurring to the e-ph interaction can qualitatively account for the values of $\lambda$ and indicate which Fermi level transitions contribute most.

In $\mathrm{Bi}_{2} \mathrm{Se}_{3}(111)$ the Fermi level cuts the Dirac cone arising from the Dirac point, as well as pairs of topological quantum-well states and the bottom of the conduction band; in $\mathrm{Bi}_{2} \mathrm{Te}_{3}(111)$ the Dirac cone and apparently only the conduction-band bottom; in $\mathrm{Bi}_{2} \mathrm{Te}_{2} \mathrm{Se}(111)$ only the Dirac states, in $\mathrm{Sb}_{2} \mathrm{Te}_{3}(111)$, where the Dirac point is above the Fermi level and there are altogether six hole pockets (three spin-up and three spin-down, as indicated by arrows in Figure 8c), there is an intervalley e-ph coupling, in addition to the contribution from Dirac holes. It appears that the Dirac states alone contribute little to the total e-ph interaction, and that larger contributions come from the surface quantum-well states and the bottom of the conduction band at the surface. The observation that $\lambda_{H A S}$ increases when quantum-well and conduction-band states are involved agrees with the result of DFPT calculations of $\lambda$ as a function of the Fermi level position recently reported by Heid et al. [119]. The above values of $\lambda_{H A S}$ are also in agreement with the corresponding values from other sources: for $\mathrm{Bi}_{2} \mathrm{Se}_{3}(111) \lambda=0.17$ [120], 0.25 [121], 0.26 [122]; for $\mathrm{Bi}_{2} \mathrm{Te}_{3}(111) \lambda=0.19$ [120], for $\mathrm{Bi}_{2} \mathrm{Te}_{2} \mathrm{Se}(111) \lambda=0.12$ [15]. No other value of $\lambda$ to compare with $\lambda_{H A S}$ is available, to our knowledge, for $\mathrm{Sb}_{2} \mathrm{Te}_{3}(111)$, though it is noted in [123] that in $\mathrm{Sb}_{2} \mathrm{Te}_{3}$ the e-ph interaction from ab-initio calculations is weaker than in $\mathrm{Bi}_{2} \mathrm{Te}_{3}$, as actually found here. 

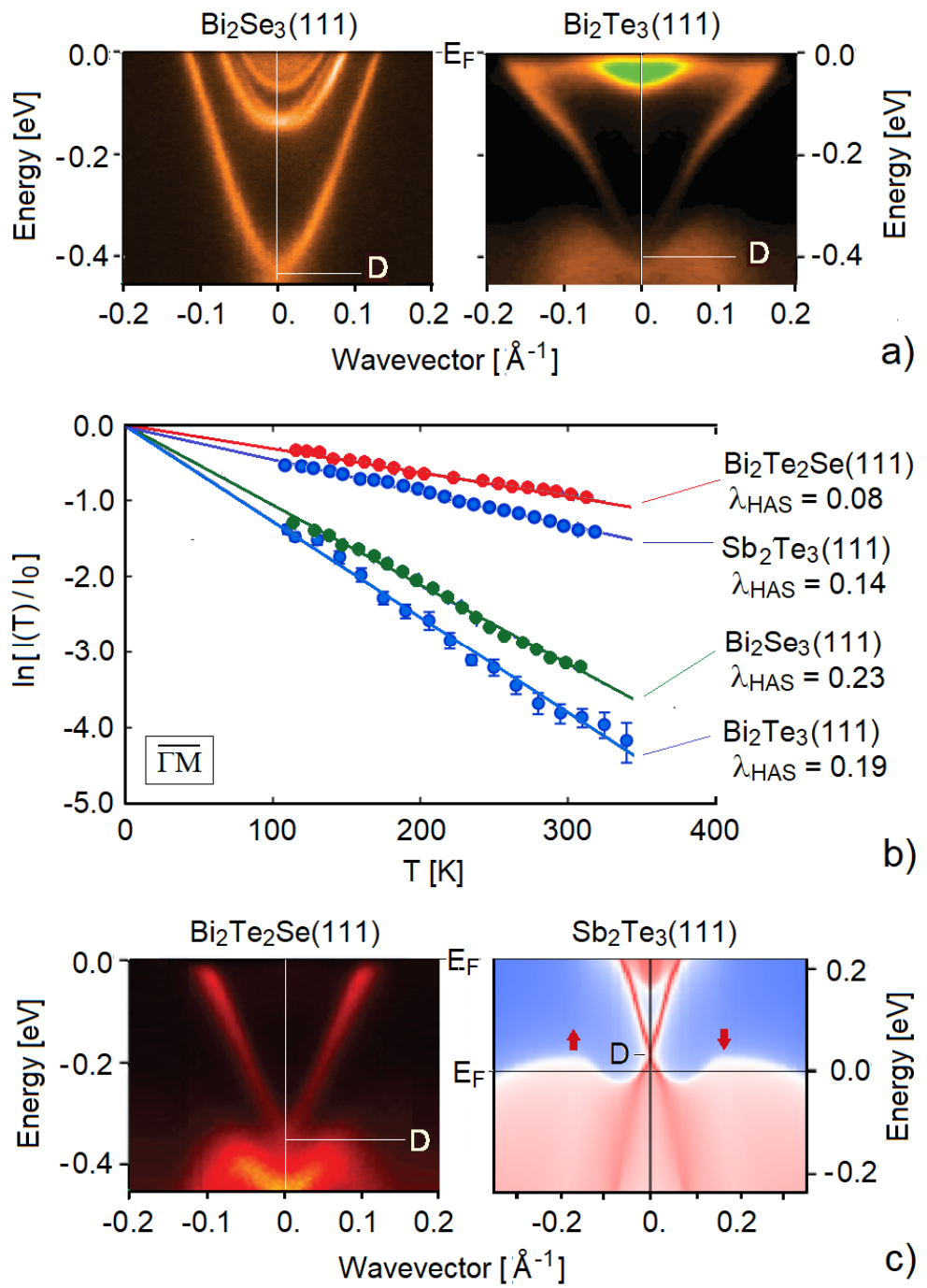

Figure 8. ARPES data for the (111) surface of n-type pnictogen chalcogenides: (a) $\mathrm{Bi}_{2} \mathrm{Se}_{3}$ and $\mathrm{Bi}_{2} \mathrm{Te}_{3}$ and (c) $\mathrm{Bi}_{2} \mathrm{Te}_{2} \mathrm{Se}$, with decreasing binding energy of the Dirac point (D) and of the surface conduction-band minimum (from $0.15 \mathrm{eV}$ in $\mathrm{Bi}_{2} \mathrm{Se}_{3}$ to $0.08 \mathrm{eV}$ in $\mathrm{Bi}_{2} \mathrm{Te}_{3}$ and $\approx 0$ in $\mathrm{Bi}_{2} \mathrm{Te}_{2} \mathrm{Se}$ (adapted from [15] published 2020 by the American Chemical Society)). For comparison ab-initio calculations of the surface band structure of $\mathrm{Sb}_{2} \mathrm{Te}_{3}$ (111) [118], where in addition to the Dirac cone, hole pockets of opposite spin are also found (upward and downward arrows). (b) The DW exponent slopes obtained from HAS specular intensity measured as functions of temperature with the scattering plane in the $\overline{\Gamma M}$ direction for the four samples. The corresponding e-ph coupling constants $\lambda \equiv \lambda_{H A S}$ decrease from $\mathrm{Bi}_{2} \mathrm{Se}_{3}(111)$ to $\mathrm{Bi}_{2} \mathrm{Te}_{2} \mathrm{Se}(111)$, suggesting a dominant role in the e-ph interaction of the conduction-band quantum-well electronic states over the Dirac electrons and the intervalley transitions.

\section{Conclusions}

In this brief review HAS from conducting surfaces has been shown to provide quantitative information on the electron-phonon (e-ph) coupling constants in some classes of 2D superconductors. Although the temperature dependence of HAS reflectivity, expressed via the DW exponent, allows for the determination of the global e-ph interaction through the mass-enhancement factor $\lambda$, inelastic HAS intensities from single phonons turn out to be proportional to the mode-selected e-ph coupling constants $\lambda_{\mathbf{Q} v}$ (mode-lambda spectroscopy). The latter possibility has been illustrated by the study of $\mathrm{Pb}$ ultra-thin films of 5 and 7 monolayers, while the derivation of $\lambda$ from the temperature dependence of the DW exponent has been discussed for Pb films 1 to 24 ML thick, as well as for a high-index, 
quasi-1D bismuth surface and for superconducting layered chalcogenides. Concerning transition-metal dichalcogenides, an important aspect that HAS spectroscopy can help elucidate is whether and where the coexistence of CDWs and superconductivity is competitive or cooperative. Pnictogen chalcogenides, where topological superconductivity can be induced by pressure, intercalation, or proximity to superconductors, offer possible scenarios of coexistence with CDWs, after the recent observation of CDWs in some of these materials. The values of $\lambda$ obtained by HAS in $n$-type Bi chalcogenides permit the appreciation of the role of surface quantum well and conduction-band states with respect to that of Dirac states.

In view of these potentialities it would be desirable to investigate by HAS various classes of high- $T_{\mathcal{C}}$ superconductors, in particular cuprates. No HAS study addressing e-ph interaction has been carried out so far in these materials. E-ph interaction in perovskites has been shown in the 1980s by Heinz Bilz and his school to receive an important contribution from the non-linear polarizability of oxygen ions [124], and to be therefore responsible for ferroelectricity [125-129] and other structural phase transitions $[130,131]$, with possible implications for high- $T_{\mathcal{C}}$ superconductivity in perovskites [131]. Given the layered structure of these materials and the good quality of their surfaces, high-resolution HAS studies of their complex phonon structure and of mode-selected e-ph coupling constants would certainly help to further advance the understanding of high- $T_{\mathcal{C}}$ superconductivity. The simultaneous information on dynamics and structure of the surface electron density that can be obtained with HAS would also be instrumental in investigating certain aspects of high- $T_{\mathcal{c}}$ materials, e.g., phase separation [132], heterogeneity [133,134] and the superstripes landscape [135-137] made of multiscale puddles of CDWs from $3 \mathrm{~nm}$ to hundreds of $\mathrm{nm}$ [138] controlled by doping and elastic strain [139]. The nanoscale CDW texturing has been observed in 1T-TiSe ${ }_{2}$ [140] where by Ti self-doping the Fermi level is tuned near a Lifshitz transition in correlated multi-band systems as was predicted theoretically [141]. Moreover, it has been confirmed that the CDW texture in $\mathrm{Sr}$ doped $\mathrm{Bi}_{2} \mathrm{Se}_{3}$ [142] can be manipulated by uniaxial strain to control superconductivity.

Inelastic HAS spectroscopy, besides measuring single surface phonon frequencies and the related $\lambda_{\mathbf{Q} v}$, allows for the observation of low-energy collective electronic excitations like phasons, amplitons and acoustic surface plasmons (ASP) $[1,19,20,143]$ due to the direct "mechanical" interaction (Pauli repulsion) of He atoms with the surface electron density. When the ASP dispersion curve (linear in the long-wavelength limit [144]) enters the surface-projected phonon density, there are avoided crossings with the surface phonon dispersion curves and a robust renormalization of ASP phase velocity, all driven by e-ph interaction. Actually, a strong ASP-phonon interaction is at the basis of a recent prediction by Shvonski et al. [145] of polaron plasmons as possible hybrid excitations at topological metal surfaces, reviving the old concept by Lemmens and Devreese [146] of collective excitations of the polaron gas. In view of the importance that has been attributed to polaron pairing (bipolarons) in the early days of high- $T_{\mathcal{C}}$ superconductivity [147-150], the strong e-ph coupling in cuprates has been confirmed experimentally by the anomalous doping dependent isotope effect: (i) on the superconductivity critical temperature [151], which shows the proximity of the chemical potential to a Lifshitz transition at 1/8 doping [152], (ii) on the polaronic CDW ordering temperature $T^{*}$ [153] and (iii) the kink in the electronic dispersion from angle-resolved photoemission spectra [154]. Therefore, the study of exotic collective polaron excitations in topological superconductors by HAS could have many surprises in store.

Author Contributions: G.B., J.R.M., S.M.-A. and J.P.T. developed the physical interpretation of the data. A.T. and A.R. performed the experimental measurements in terms of the topological insulator and semimetal surfaces together with most of the data analysis concerned with these samples. G.B. wrote the paper, and all authors discussed the results and contributed to the preparation of the manuscript. All authors have read and agreed to the published version of the manuscript.

Funding: A.R., W.E.E., and A.T. acknowledge financial support provided by the FWF (Austrian Science Fund) within the projects J3479-N20 and P29641-N36. 
Acknowledgments: This work is dedicated to Alex Müller with profound admiration for having opened new horizons in modern physics with his discovery, with Georg Bednorz, of high-temperature superconductivity. GB is particular grateful to Alex for the privilege of having been a witness of the early days of high-Tc superconductivity at the Ettore Majorana Foundation and Centre for Scientific Culture (EMFCSC) in Erice (Sicily), and for the stimulus to start, with his active participation and the collaboration of the present Festschrift editors, a series of periodic courses on superconductivity (now continued by Antonio Bianconi) within the International School of Solid State Physics at EMFCSC [155]. We thank Martin Bremholm, Philip Hofmann and co-workers for the synthesis of the topological insulator samples and providing us with the Bi(114) sample.

Conflicts of Interest: The authors declare no conflict of interest.

\section{References}

1. Benedek, G.; Toennies, J.P. 2018 Atomic-Scale Dynamics at Surfaces-Theory and Experimental Studies with Helium Atom. Scattering; Springer: Berlin, Germany, 2018.

2. Doak, R.B.; Harten, U.; Toennies, J.P. Anomalous Surface Phonon Dispersion Relations for Ag(111) Measured by Inelastic Scattering of He. Atoms Phys. Rev. Lett. 1983, 51, 578-581. [CrossRef]

3. Harten, U.; Toennies, J.P.; Wöll, C. Helium time-of-flight spectroscopy of surface-phonon dispersion curves of the noble metals. Faraday Discuss. Chem. Soc. 1985, 80, 137-149. [CrossRef]

4. Jayanthi, C.S.; Bilz, H.; Kress, W.; Benedek, G. Nature of surface-phonon anomalies in noble metals. Phys. Rev. Lett. 1987, 59, 795-798. [CrossRef] [PubMed]

5. Kaden, C.; Ruggerone, P.; Toennies, J.P.; Zhang, G.; Benedek, G. Electronic pseudocharge model for the $\mathrm{Cu}(111)$ longitudinal-surface-phonon anomaly observed by helium-atom scattering. Phys. Rev. B 1992, 46, 13509-13525. [CrossRef] [PubMed]

6. Benedek, G.; Bernasconi, M.; Chis, V.; Chulkov, E.; Echenique, P.M.; Hellsing, B.; Toennies, J.P. Theory of surface phonons at metal surfaces: Recent advances. J. Phys. Condens. Matter 2010, 22, 084020. [CrossRef] [PubMed]

7. Sklyadneva, I.Y.; Benedek, G.; Chulkov, E.V.; Echenique, P.M.; Heid, R.; Bohnen, K.-P.; Toennies, J.P. Mode-Selected Electron-Phonon Coupling in Superconducting Pb Nanofilms Determined from He Atom Scattering. Phys. Rev. Lett. 2011, 107, 095502. [CrossRef]

8. Tamtögl, A.; Ruckhofer, A.; Campi, D.; Allison, W.; Ernst, W.E. Atom-surface van der Waals Potentials of Topological Insulators and Semimetals from Scattering Measurements. Phys. Chem. Chem. Phys. 2020, in press.

9. Benedek, G.; Bernasconi, M.; Bohnen, K.-P.; Campi, D.; Chulkov, E.V.; Echenique, P.M.; Heid, R.; Sklyadneva, I.Y.; Toennies, J.P. Unveiling mode-selected electron-phonon interactions in metal films by helium atom scattering. Phys. Chem. Chem. Phys. 2014, 16, 7159-7172. [CrossRef]

10. Manson, J.R.; Benedek, G.; Miret-Artés, S. Electron-Phonon Coupling Strength at Metal Surfaces Directly Determined from the Helium Atom Scattering Debye-Waller Factor. J. Phys. Chem. Lett. 2016, 7, 1016-1021. [CrossRef]

11. Benedek, G.; Miret-Artés, S.; Toennies, J.P.; Manson, J.R. Electron-Phonon Coupling Constant of Metallic Overlayers from Specular He Atom Scattering. J. Phys. Chem. Lett. 2018, 9, 76-83. [CrossRef]

12. Anemone, G.; Taleb, A.A.; Benedek, G.; Castellanos-Gomez, A.; Farías, D. Electron-Phonon Coupling Constant of $2 \mathrm{H}-\mathrm{MoS}_{2}(0001)$ from Helium-Atom Scattering. J. Phys. Chem. C 2019, 123, 3682-3686. [CrossRef]

13. Anemone, G.; Garnica, M.; Zappia, M.; Aguilar, P.C.; Taleb, A.A.; Kuo, C.-N.; Lue, C.S.; Politano, A.; Benedek, G.; Parga, A.L.V.; et al. Experimental determination of surface thermal expansion and electron-phonon coupling constant of 1T-PtTe 2. 2D Mater. 2020, 7, 025007.

14. Tamtögl, A.; Kraus, P.; Mayrhofer-Reinhartshuber, M.; Benedek, G.; Bernasconi, M.; Dragoni, D.; Campi, D.; Ernst, W.E. Statics and dynamics of multivalley charge density waves in Sb(111). npj Quantum Mater. 2019, 4, 28. [CrossRef]

15. Benedek, G.; Miret-Artés, S.; Manson, J.R.; Ruckhofer, A.; Ernst, W.E.; Tamtögl, A. Origin of the Electron-Phonon Interaction of Topological Semimetal Surfaces Measured with Helium Atom Scattering. J. Phys. Chem. Lett. 2020, 11, 1927-1933. [CrossRef]

16. Hofmann, P.; Ugeda, M.M.; Tamtögl, A.; Ruckhofer, A.; Ernst, W.E.; Benedek, G.; Martínez-Galera, A.J.; Stróżecka, A.; Gómez-Rodríguez, J.M.; Rienks, E.; et al. Strong-coupling charge density wave in a one-dimensional topological metal. Phys. Rev. B 2019, 99, 035438. [CrossRef] 
17. Benedek, G.; Manson, J.R.; Miret-Artés, S. The Electron-Phonon Interaction of Low-Dimensional and Multi-Dimensional Materials from He Atom Scattering. Adv. Mater. 2020, 32, 2002072. [CrossRef]

18. Benedek, G.; Manson, J.R.; Miret-Artés, S. The electron-phonon coupling constant for single-layer graphene on metal substrates determined from He atom scattering. Phys. Chem. Chem. Phys. 2020, in press. [CrossRef]

19. Ruckhofer, A.; Campi, D.; Bremholm, M.; Hofmann, P.; Benedek, G.; Bernasconi, M.; Ernst, W.E.; Tamtögl, A. Terahertz surface modes and electron-phonon coupling on $\mathrm{Bi}_{2} \mathrm{Se}_{3}(111)$. Phys. Rev. Res. 2020, 2, 023186. [CrossRef]

20. Ruckhofer, A.; Halbritter, S.E.; Lund, H.; Holt, A.J.U.; Bianchi, M.; Bremholm, M.; Benedek, G.; Hofmann, P.; Ernst, W.E.; Tamtögl, A. Inelastic helium atom scattering from $\mathrm{Sb}_{2} \mathrm{Te}_{3}(111)$ : Phonon dispersion, focusing effects and surfing. Phys. Chem. Chem. Phys. 2020, in press. [CrossRef]

21. Esbjerg, N.; Nørskov, J.K. Dependence of the He-Scattering Potential at Surfaces on the Surface-Electron-Density Profile. Phys. Rev. Lett. 1980, 45, 807-810. [CrossRef]

22. Cole, M.W.; Toigo, F. Energy of immersing a $\mathrm{He}, \mathrm{Ne}$, or Ar atom or $\mathrm{H}_{2}$ molecule into a low-density electron gas. Phys. Rev. B 1985, 31, 727-729. [CrossRef] [PubMed]

23. Manson, R.; Celli, V. Inelastic surface scattering of non-penetrating particles. Surf. Sci. 1971, 24, 495-514. [CrossRef]

24. Grimvall, G. The Electron-Phonon Interaction in Normal. Metals Phys. Scr. 1976, 14, 63. [CrossRef]

25. Allen, P.B. The Electron-Phonon Coupling Constant $\lambda$. In Handbook of Superconductivity; Poole, C.P., Jr., Ed.; Academic Press: New York, NY, USA, 1999; pp. 478-483.

26. Hinch, B.J.; Koziol, C.; Toennies, J.P.; Zhang, G. Evidence for Quantum Size Effects Observed by Helium Atom Scattering during the Growth of $\mathrm{Pb}$ on $\mathrm{Cu}(111)$. Europhys. Lett. EPL 1989, 10, 341-346. [CrossRef]

27. Zhang, G. Max-Planck-Institut für Strömungsforschung. Ph.D. Thesis, University of Göttingen, Göttingen, Germany, 1991.

28. Braun, J.; Ruggerone, P.; Zhang, G.; Toennies, J.P.; Benedek, G. Surface phonon dispersion curves of thin Pb films on $\mathrm{Cu}(111)$. Phys. Rev. B 2009, 79, 205423. [CrossRef]

29. Guo, Y.; Zhang, Y.-F.; Bao, X.-Y.; Han, T.-Z.; Tang, Z.; Zhang, L.-X.; Zhu, W.-G.; Wang, E.G.; Niu, Q.; Qiu, Z.Q.; et al. Superconductivity Modulated by Quantum Size Effects. Science 2004, 306, 1915-1917. [CrossRef]

30. Eom, D.; Qin, S.; Chou, M.-Y.; Shih, C.K. Persistent Superconductivity in Ultrathin Pb Films: A Scanning Tunneling Spectroscopy Study. Phys. Rev. Lett. 2006, 96, 027005. [CrossRef]

31. Özer, M.M.; Jia, Y.; Zhang, Z.; Thompson, J.R.; Weitering, H.H. Tuning the Quantum Stability and Superconductivity of Ultrathin Metal Alloys. Science 2007, 316, 1594-1597. [CrossRef]

32. Qin, S.; Kim, J.; Niu, Q.; Shih, C.-K. Superconductivity at the Two-Dimensional Limit. Science 2009, 324, 1314-1317. [CrossRef]

33. Brun, C.; Hong, I.-P.; Patthey, F.; Sklyadneva, I.Y.; Heid, R.; Echenique, P.M.; Bohnen, K.P.; Chulkov, E.V.; Schneider, W.-D. Reduction of the Superconducting Gap of Ultrathin Pb Islands Grown on Si(111). Phys. Rev. Lett. 2009, 102. [CrossRef]

34. Zhang, T.; Cheng, P.; Li, W.-J.; Sun, Y.-J.; Wang, G.; Zhu, X.-G.; He, K.; Wang, L.; Ma, X.; Chen, X.; et al. Superconductivity in one-atomic-layer metal films grown on $\mathrm{Si}(111)$. Nat. Phys. 2010, 6, 104-108. [CrossRef]

35. Zhang, Y.-F.; Jia, J.-F.; Han, T.-Z.; Tang, Z.; Shen, Q.-T.; Guo, Y.; Qiu, Z.Q.; Xue, Q.-K. Band Structure and Oscillatory Electron-Phonon Coupling of $\mathrm{Pb}$ Thin Films Determined by Atomic-Layer-Resolved Quantum-Well States. Phys. Rev. Lett. 2005, 95, 096802. [CrossRef] [PubMed]

36. Prakash, O.; Kumar, A.; Thamizhavel, A.; Ramakrishnan, S. Evidence for bulk superconductivity in pure bismuth single crystals at ambient pressure. Science 2017, 355, 52-55. [CrossRef] [PubMed]

37. Mata-Pinzón, Z.; Valladares, A.A.; Valladares, R.M.; Valladares, A. Superconductivity in Bismuth. A New Look at an Old Problem. PLoS ONE 2016, 11, e0147645.

38. Tian, M.; Wang, J.; Ning, W.; Mallouk, T.E.; Chan, M.H.W. Surface Superconductivity in Thin Cylindrical Bi Nanowire. Nano Lett. 2015, 15, 1487-1492. [CrossRef]

39. Wells, J.W.; Dil, J.H.; Meier, F.; Lobo-Checa, J.; Petrov, V.N.; Osterwalder, J.; Ugeda, M.M.; Fernandez-Torrente, I.; Pascual, J.I.; Rienks, E.D.L.; et al. Nondegenerate Metallic States on Bi(114): A One-Dimensional Topological Metal. Phys. Rev. Lett. 2009, 102, 096802. [CrossRef]

40. Ma, T.; Wang, S. Phase Transition Dynamics; Springer: New York, NY, USA, 2013.

41. Liu, R.; Ma, T.; Wang, S.; Yang, J. Dynamic Theory of Fluctuations and Critical Exponents of Thermodynamic Phase Transitions. 2019. Available online: https://hal.archives-ouvertes.fr/hal-01674269 (accessed on 2 December 2020). 
42. Brusdeylins, G.; Heimlich, C.; Skofronick, J.G.; Toennies, J.P.; Vollmer, R.; Benedek, G. Determination of the Critical Exponent for a Charge Density Wave Transition in $2 \mathrm{H}-\mathrm{TaSe}_{2}$ by Helium Atom Scattering. Europhys. Lett. 1989, 9, 563-568. [CrossRef]

43. Brusdeylins, G.; Heimlich, C.; Skofronick, J.G.; Toennies, J.P.; Vollmer, R.; Benedek, G.; Miglio, L. He-atom scattering study of the temperature-dependent charge-density-wave surface structure and lattice dynamics of $2 \mathrm{H}-\mathrm{TaSe}_{2}(001)$. Phys. Rev. B 1990, 41, 5707-5716. [CrossRef]

44. Requardt, H.; Kalning, M.; Burandt, B.; Press, W.; Currat, R. Critical x-ray scattering at the Peierls transition in the quasi-one-dimensional system. J. Phys. Condens. Matter 1996, 8, 2327-2336. [CrossRef]

45. Lorenzo, J.E.; Currat, R.; Monceau, P.; Hennion, B.; Berger, H.; Levy, F. A neutron scattering study of the quasi-one-dimensional conductor. J. Phys. Condens. Matter 1998, 10, 5039-5068. [CrossRef]

46. Wilson, J.A.; Salvo, F.J.D.; Mahajan, S. Charge-density waves and superlattices in the metallic layered transition metal dichalcogenides. Adv. Phys. 1975, 24, 117-201. [CrossRef]

47. Nagata, S.; Aochi, T.; Abe, T.; Ebisu, S.; Hagino, T.; Seki, Y.; Tsutsumi, K. Superconductivity in the layered compound 2H-TaS 2 . J. Phys. Chem. Solids 1992, 53, 1259-1263. [CrossRef]

48. Sipos, B.; Kusmartseva, A.F.; Akrap, A.; Berger, H.; Forró, L.; Tutiš, E. From Mott state to superconductivity in $1 \mathrm{~T}-\mathrm{TaS}_{2}$. Nat. Mater. 2008, 7, 960-965. [CrossRef] [PubMed]

49. Navarro-Moratalla, E.; Island, J.O.; Mañas-Valero, S.; Pinilla-Cienfuegos, E.; Castellanos-Gomez, A.; Quereda, J.; Rubio-Bollinger, G.; Chirolli, L.; Silva-Guillén, J.A.; Agraït, N.; et al. Enhanced superconductivity in atomically thin $\mathrm{TaS}_{2}$. Nat. Commun. 2016, 7, 11043. [CrossRef]

50. Yang, Y.; Fang, S.; Fatemi, V.; Ruhman, J.; Navarro-Moratalla, E.; Watanabe, K.; Taniguchi, T.; Kaxiras, E.; Jarillo-Herrero, P. Enhanced superconductivity upon weakening of charge density wave transport in $2 \mathrm{H}-\mathrm{TaS}_{2}$ in the two-dimensional limit. Phys. Rev. B 2018, 98, 035203. [CrossRef]

51. Peng, J.; Yu, Z.; Wu, J.; Zhou, Y.; Guo, Y.; Li, Z.; Zhao, J.; Wu, C.; Xie, Y. Disorder Enhanced Superconductivity toward $\mathrm{TaS}_{2}$ Monolayer. ACS Nano 2018, 12, 9461-9466. [CrossRef]

52. Ribak, A.; Skiff, R.M.; Mograbi, M.; Rout, P.K.; Fischer, M.H.; Ruhman, J.; Chashka, K.; Dagan, Y.; Kanigel, A. Chiral superconductivity in the alternate stacking compound $4 \mathrm{Hb}-\mathrm{TaS}_{2}$. Sci. Adv. 2020, 6, eaax9480. [CrossRef]

53. Ritschel, T.; Trinckauf, J.; Garbarino, G.; Hanfland, M.; Zimmermann, M.V.; Berger, H.; Büchner, B.; Geck, J. Pressure dependence of the charge density wave in $1 \mathrm{~T}-\mathrm{TaS}_{2}$ and its relation to superconductivity. Phys. Rev. B 2013, 87, 125135. [CrossRef]

54. Wagner, K.E.; Morosan, E.; Hor, Y.S.; Tao, J.; Zhu, Y.; Sanders, T.; McQueen, T.M.; Zandbergen, H.W.; Williams, A.J.; West, D.V.; et al. Tuning the charge density wave and superconductivity in $\mathrm{Cu}_{\mathrm{x}} \mathrm{TaS}_{2}$. Phys. Rev. B 2008, 78, 104520. [CrossRef]

55. Wilson, J.A.; Salvo, F.J.D.; Mahajan, S. Charge-density waves and superlattices in the metallic layered transition metal dichalcogenides. Adv. Phys. 2001, 50, 1171-1248. [CrossRef]

56. Coleman, R.V.; McNairy, W.W.; Slough, C.G. Amplitude modulation of charge-density-wave domains in $1 \mathrm{~T}-\mathrm{TaS}_{2}$ at $300 \mathrm{~K}$. Phys. Rev. B 1992, 45, 1428-1431. [CrossRef] [PubMed]

57. Yu, Y.; Yang, F.; Lu, X.F.; Yan, Y.J.; Cho, Y.-H.; Ma, L.; Niu, X.; Kim, S.; Son, Y.-W.; Feng, D.; et al. Gate-tunable phase transitions in thin flakes of 1T-TaS 2 . Nanotechnology 2015, 10, 270-276. [CrossRef] [PubMed]

58. Cantini, P.; Boato, G.; Colella, R. Surface charge density waves observed by atomic beam diffraction. Physica B+C 1980, 99, 59-63. [CrossRef]

59. Heimlich, C. Max-Planck Inst. für Strömungsforschung. Ph.D. Thesis, University of Göttingen, Göttingen, Germany, October 1987.

60. Brusdeylins, G.; Heimlich, C.; Toennies, J.P. Helium scattering from the layered compound single crystal surface of $1 \mathrm{~T}-\mathrm{TaS}_{2}$ in the temperature region of charge density wave reconstruction. Surf. Sci. 1989, 211-212, 98-105. [CrossRef]

61. Shimada, T.; Ohuchi, F.S.; Parkinson, B.A. Work Function and Photothreshold of Layered Metal Dichalcogenides. Jpn. J. Appl. Phys. 1994, 33. [CrossRef]

62. Zhao, R.; Grisafe, B.; Ghosh, R.K.; Holoviak, S.; Wang, B.; Wang, K.; Briggs, N.; Haque, A.; Datta, S.; Robinson, J. Two-dimensional tantalum disulfide: Controlling structure and properties via synthesis. 2D Materials 2018, 5, 025001. [CrossRef]

63. Rossnagel, K. On the origin of charge-density waves in select layered transition-metal dichalcogenides. J. Phys. Condens. Matter. 2011, 23, 213001. [CrossRef] 
64. Shiino, O.; Watanabe, T.; Endo, T.; Hanaguri, T.; Kitazawa, K.; Nohara, M.; Takagi, H.; Murayama, C.; Takeshita, N.; Môri, N.; et al. Metal-insulator transition in $1 \mathrm{~T}-\mathrm{TaS}_{2-\mathrm{x}} \mathrm{Se}_{\mathrm{x}}$. Phys. B Condens. Matter 2000, 284-288, 1673-1674. [CrossRef]

65. Balaguru-Rayappan, J.B.; Raj, S.A.C.; Lawrence, N. Thermal properties of $1 \mathrm{~T}-\mathrm{TaS}_{2}$ at the onset of charge density wave states. Phys. B Condens. Matter. 2010, 405, 3172-3175. [CrossRef]

66. Liu, A.Y. Electron-phonon coupling in compressed $1 \mathrm{~T}-\mathrm{TaS}_{2}$ : Stability and superconductivity from first principles. Phys. Rev. B 2009, 79, 220515. [CrossRef]

67. Benedek, G.; Hofmann, F.; Ruggerone, P.; Onida, G.; Miglio, L. Surface phonons in layered crystals: Theoretical aspects. Surf. Sci. Rep. 1994, 20,1-43. [CrossRef]

68. Benedek, G.; Brusdeylins, G.; Hofmann, F.; Ruggerone, P.; Toennies, J.P.; Vollmer, R.; Skofronick, J.G. Strong coupling of Rayleigh phonons to charge density waves in 1T-TaS 2 . Surf. Sci. 1994, 304, 185-190. [CrossRef]

69. McWhan, D.B.; Axe, J.D.; Youngblood, R. Pressure dependence of the striped-to-hexagonal charge-density-wave transition in 2H-TaSe2. Phys. Rev. B 1981, 24, 5391-5393. [CrossRef]

70. Tsoutsou, D.; Aretouli, K.E.; Tsipas, P.; Marquez-Velasco, J.; Xenogiannopoulou, E.; Kelaidis, N.; Aminalragia-Giamini, S.; Dimoulas, A. Epitaxial 2D MoSe $2\left(\mathrm{HfSe}_{2}\right)$ Semiconductor/2D TaSe 2 Metal van der Waals Heterostructures. ACS Appl. Mater. Interfaces 2016, 8, 1836-1841. [CrossRef]

71. Moncton, D.E.; Axe, J.D.; DiSalvo, F.J. Neutron scattering study of the charge-density wave transitions in $2 \mathrm{H}-\mathrm{TaSe}_{2}$ and $2 \mathrm{H}-\mathrm{NbSe}_{2}$. Phys. Rev. B 1977, 16, 801-819. [CrossRef]

72. Craven, R.A.; Meyer, S.F. Specific heat and resistivity near the charge-density-wave phase transitions in 2H-TaSe 2 and 2H-TaS 2 . Phys. Rev. B 1977, 16, 4583-4593. [CrossRef]

73. Bhoi, D.; Khim, S.; Nam, W.; Lee, B.S.; Kim, C.; Jeon, B.-G.; Min, B.H.; Park, S.; Kim, K.H. Interplay of charge density wave and multiband superconductivity in $2 \mathrm{H}-\mathrm{Pd}_{\mathrm{x}} \mathrm{TaSe}_{2}$. Sci. Rep. 2016, 6, 24068. [CrossRef]

74. Luo, H.; Xie, W.; Tao, J.; Inoue, H.; Gyenis, A.; Krizan, J.W.; Yazdani, A.; Zhu, Y.; Cava, R.J. Polytypism, polymorphism, and superconductivity in $\mathrm{TaSe}_{2-\mathrm{x}} \mathrm{Te}_{\mathrm{x}}$. Proc. Natl. Acad. Sci. USA 2015, 112, E1174-E1180. [CrossRef]

75. Kumakura, T.; Tan, H.; Handa, T.; Morishita, M.; Fukuyama, H. Charge density waves and superconductivity in $2 \mathrm{H}-\mathrm{TaSe}_{2}$. Czechoslov. J. Phys. 1996, 46, 2611-2612. [CrossRef]

76. Yokota, K.; Kurata, G.; Matsui, T.; Fukuyama, H. Superconductivity in the quasi-two-dimensional conductor 2H-TaSe 2 . Phys. B Condens. Matter. 2000, 284-288, 551-552. [CrossRef]

77. Lian, C.-S.; Heil, C.; Liu, X.; Si, C.; Giustino, F.; Duan, W. Coexistence of Superconductivity with Enhanced Charge Density Wave Order in the Two-Dimensional Limit of TaSe 2 . J. Phys. Chem. Lett. 2019, 10, 4076-4081. [CrossRef] [PubMed]

78. Wu, Y.; He, J.; Liu, J.; Xing, H.; Mao, Z.; Liu, Y. Dimensional reduction and ionic gating induced enhancement of superconductivity in atomically thin crystals of $2 \mathrm{H}-\mathrm{TaSe}_{2}$. Nanotechnology 2018, 30, 035702. [CrossRef] [PubMed]

79. Liu, Y.; Shao, D.F.; Li, L.J.; Lu, W.J.; Zhu, X.D.; Tong, P.; Xiao, R.C.; Ling, L.S.; Xi, C.Y.; Pi, L.; et al. Nature of charge density waves and superconductivity in $1 \mathrm{~T}-\mathrm{TaSe}_{2-\mathrm{x}} \mathrm{Te}_{\mathrm{x}}$. Phys. Rev. B 2016, 94, 045131. [CrossRef]

80. Li, X.C.; Zhou, M.H.; Yang, L.H.; Dong, C. Significant enhancement of superconductivity in copper-doped 2H-TaSe 2 . Supercond. Sci. Technol. 2017, 30, 125001. [CrossRef]

81. Benedek, G. Summary Abstract: Surface phonon dynamics of 2H-TaSe 2 (001). J. Vac. Sci. Technol. A 1987, 5, 1093-1094. [CrossRef]

82. Benedek, G.; Brusdeylins, G.; Heimlich, C.; Miglio, L.; Skofronick, J.G.; Toennies, J.P.; Vollmer, R. Shifted surface-phonon anomaly in $2 \mathrm{H}-\mathrm{TaSe}_{2}$. Phys. Rev. Lett. 1988, 60, 1037-1040. [CrossRef] [PubMed]

83. Hulpke, E. Helium Atom Scattering from Surfaces; Springer: Berlin, Germany, 1992.

84. Murphy, B.M.; Requardt, H.; Stettner, J.; Serrano, J.; Krisch, M.; Müller, M.; Press, W. Phonon Modes at the $2 \mathrm{H}-\mathrm{NbSe}_{2}$ Surface Observed by Grazing Incidence Inelastic X-Ray Scattering. Phys. Rev. Lett. 2005, 95, 256104. [CrossRef]

85. Weber, F.; Rosenkranz, S.; Castellan, J.-P.; Osborn, R.; Hott, R.; Heid, R.; Bohnen, K.-P.; Egami, T.; Said, A.H.; Reznik, D. Extended Phonon Collapse and the Origin of the Charge-Density Wave in $2 \mathrm{H}-\mathrm{NbSe}_{2}$. Phys. Rev. Lett. 2011, 107, 107403. [CrossRef]

86. Anemone, G.; Casado Aguilar, M.P.; Garnica, A.; Al Taleb, C.-N.; Kuo, C.; Shan-Lue, A.; Politano, A.L.; Vàzquez de Parga, G.; Benedek, D.; Farìas, R.M. Electron-Phonon Coupling in Superconducting 1T-PdTe2. To be publish. 
87. Hor, Y.S.; Williams, A.J.; Checkelsky, J.G.; Roushan, P.; Seo, J.; Xu, Q.; Zandbergen, H.W.; Yazdani, A.; Ong, N.P.; Cava, R.J. Superconductivity in $\mathrm{Cu}_{\mathrm{x}} \mathrm{Bi}_{2} \mathrm{Se}_{3}$ and its Implications for Pairing in the Undoped Topological Insulator. Phys. Rev. Lett. 2010, 104, 057001. [CrossRef]

88. Hor, Y.S.; Checkelsky, J.G.; Qu, D.; Ong, N.P.; Cava, R.J. Superconductivity and non-metallicity induced by doping the topological insulators $\mathrm{Bi}_{2} \mathrm{Se}_{3}$ and $\mathrm{Bi}_{2} \mathrm{Te}_{3}$. J. Phys. Chem. Solids 2011, 72, 572-576. [CrossRef]

89. Bray-Ali, N. Anon How to turn a topological insulator into a superconductor. Physics 2010, 3, 11. [CrossRef]

90. Yonezawa, S. Nematic Superconductivity in Doped $\mathrm{Bi}_{2} \mathrm{Se}_{3}$ Topological Superconductors. Condens. Matter. 2019, 4, 2. [CrossRef]

91. Zhang, H.; Li, H.; He, H.; Wang, J. Enhanced superconductivity in $\mathrm{Bi}_{2} \mathrm{Se}_{3} / \mathrm{Nb}$ heterostructures. Appl. Phys. Lett. 2019, 115, 113101. [CrossRef]

92. Matsubayashi, K.; Terai, T.; Zhou, J.S.; Uwatoko, Y. Superconductivity in the topological insulator $\mathrm{Bi}_{2} \mathrm{Te}_{3}$ under hydrostatic pressure. Phys. Rev. B 2014, 90, 125126. [CrossRef]

93. Yano, R.; Hirose, H.T.; Tsumura, K.; Yamamoto, S.; Koyanagi, M.; Kanou, M.; Kashiwaya, H.; Sasagawa, T.; Kashiwaya, S. Proximity-Induced Superconducting States of Magnetically Doped 3D Topological Insulators with High Bulk Insulation. Condens. Matter. 2019, 4, 9. [CrossRef]

94. Koren, G.; Kirzhner, T.; Lahoud, E.; Chashka, K.B.; Kanigel, A. Proximity-induced superconductivity in topological $\mathrm{Bi}_{2} \mathrm{Te}_{2} \mathrm{Se}$ and $\mathrm{Bi}_{2} \mathrm{Se}_{3}$ films: Robust zero-energy bound state possibly due to Majorana fermions. Phys. Rev. B 2011, 84, 224521. [CrossRef]

95. Qin, H.; Guo, B.; Wang, L.; Zhang, M.; Xu, B.; Shi, K.; Pan, T.; Zhou, L.; Chen, J.; Qiu, Y.; et al. Superconductivity in Single-Quintuple-Layer $\mathrm{Bi}_{2} \mathrm{Te}_{3}$ Grown on Epitaxial FeTe. Nano Lett. 2020, 20, 3160-3168. [CrossRef]

96. Charpentier, S.; Galletti, L.; Kunakova, G.; Arpaia, R.; Song, Y.; Baghdadi, R.; Wang, S.M.; Kalaboukhov, A.; Olsson, E.; Tafuri, F.; et al. Induced unconventional superconductivity on the surface states of $\mathrm{Bi}_{2} \mathrm{Te}_{3}$ topological insulator. Nat. Commun. 2017, 8, 2019. [CrossRef]

97. Kong, P.P.; Zhang, J.L.; Zhang, S.J.; Zhu, J.; Liu, Q.Q.; Yu, R.C.; Fang, Z.; Jin, C.Q.; Yang, W.G.; Yu, X.H.; et al. Superconductivity of the topological insulator $\mathrm{Bi}_{2} \mathrm{Se}_{3}$ at high pressure. J. Phys. Condens. Matter 2013, 25, 362204. [CrossRef]

98. Cai, S.; Kushwaha, S.K.; Guo, J.; Sidorov, V.A.; Le, C.; Zhou, Y.; Wang, H.; Lin, G.; Li, X.; Li, Y.; et al. Universal superconductivity phase diagram for pressurized tetradymite topological insulators. Phys. Rev. Mater. 2018, 2, 114203. [CrossRef]

99. Zhang, J.; Zhang, S.; Kong, P.; Yang, L.; Jin, C.; Liu, Q.; Wang, X.; Yu, J. Pressure induced electronic phase transitions and superconductivity in n-type $\mathrm{Bi}_{2} \mathrm{Te}_{3}$. J. Appl. Phys. 2018, 123, 125901. [CrossRef]

100. Zhang, S. Discovery of the Chiral Majorana Fermion and Its Application to Topological Quantum Computing; APS March Meeting: Nashville, TN, USA, 2018.

101. Kauffman, L.H.; Lomonaco, S.J. Braiding, Majorana fermions, Fibonacci particles and topological quantum computing. Quantum Inf. Process. 2018, 17, 201. [CrossRef]

102. Benedek, G. Majorana Fermions in Condensed Matter Scientific Papers of Ettore Majorana: A New Expanded Edition; Cifarelli, L., Ed.; Springer International Publishing: Cham, Switzerland, 2020; pp. 159-168.

103. Volovik, G.E. Fermion zero modes on vortices in chiral superconductors. J. Exp. Theor. Phys. Lett. 1999, 70, 609-614. [CrossRef]

104. Read, N.; Green, D. Paired states of fermions in two dimensions with breaking of parity and time-reversal symmetries and the fractional quantum Hall effect. Phys. Rev. B 2000, 61, 10267-10297. [CrossRef]

105. Kitaev, A. Anyons in an exactly solved model and beyond. Ann. Phys. 2006, 321, 2-111. [CrossRef]

106. Fu, L.; Kane, C.L. Superconducting Proximity Effect and Majorana Fermions at the Surface of a Topological Insulator. Phys. Rev. Lett. 2008, 100, 096407. [CrossRef]

107. Akhmerov, A.R.; Nilsson, J.; Beenakker, C.W.J. Electrically Detected Interferometry of Majorana Fermions in a Topological Insulator. Phys. Rev. Lett. 2009, 102, 216404. [CrossRef]

108. Alicea, J. New directions in the pursuit of Majorana fermions in solid state systems. Rep. Prog. Phys. 2012, 75, 076501. [CrossRef]

109. He, Q.L.; Pan, L.; Stern, A.L.; Burks, E.C.; Che, X.; Yin, G.; Wang, J.; Lian, B.; Zhou, Q.; Choi, E.S.; et al. Chiral Majorana fermion modes in a quantum anomalous Hall insulator-superconductor structure. Science 2017, 357, 294-299. [CrossRef] 
110. Xu, J.-P.; Wang, M.-X.; Liu, Z.L.; Ge, J.-F.; Yang, X.; Liu, C.; Xu, Z.A.; Guan, D.; Gao, C.L.; Qian, D.; et al. Experimental Detection of a Majorana Mode in the core of a Magnetic Vortex inside a Topological Insulator-Superconductor $\mathrm{Bi}_{2} \mathrm{Te}_{3} / \mathrm{NbSe} 2$ Heterostructure. Phys. Rev. Lett. 2015, 114, 017001. [CrossRef]

111. Lee, E.J.H.; Jiang, X.; Houzet, M.; Aguado, R.; Lieber, C.M.; De Franceschi, S. Spin-resolved Andreev levels and parity crossings in hybrid superconductor-semiconductor nanostructures. Nat. Nanotechnol. 2014, 9, 79-84. [CrossRef] [PubMed]

112. Sun, H.-H.; Zhang, K.-W.; Hu, L.-H.; Li, C.; Wang, G.-Y.; Ma, H.-Y.; Xu, Z.-A.; Gao, C.-L.; Guan, D.-D.; Li, Y.-Y.; et al. Majorana Zero Mode Detected with Spin Selective Andreev Reflection in the Vortex of a Topological Superconductor. Phys. Rev. Lett. 2016, 116, 257003. [CrossRef] [PubMed]

113. Kitaev, A. Periodic table for topological insulators and superconductors. AIP Conf. Proc. 2009, 1134, $22-30$.

114. Qi, X.-L.; Zhang, S.-C. Topological insulators and superconductors. Rev. Mod. Phys. 2011, 83, 1057-1110. [CrossRef]

115. Wang, M.-X.; Liu, C.; Xu, J.-P.; Yang, F.; Miao, L.; Yao, M.-Y.; Gao, C.L.; Shen, C.; Ma, X.; Chen, X.; et al. The Coexistence of Superconductivity and Topological Order in the $\mathrm{Bi}_{2} \mathrm{Se}_{3}$. Thin Films Sci. 2012, 336, 52-55.

116. Tamtögl, A.; Campi, D.; Bremholm, M.; Hedegaard, E.M.J.; Iversen, B.B.; Bianchi, M.; Hofmann, P.; Marzari, N.; Benedek, G.; Ellis, J.; et al. Nanoscale surface dynamics of $\mathrm{Bi}_{2} \mathrm{Te}_{3}(111)$ : Observation of a prominent surface acoustic wave and the role of van der Waals interactions. Nanoscale 2018, 10, 14627-14636. [CrossRef]

117. Tamtögl, A.; Kraus, P.; Avidor, N.; Bremholm, M.; Hedegaard, E.M.J.; Iversen, B.B.; Bianchi, M.; Hofmann, P.; Ellis, J.; Allison, W.; et al. Electron-phonon coupling and surface Debye temperature of $\mathrm{B}_{2} \mathrm{Te}_{3}(111)$ from helium atom scattering. Phys. Rev. B 2017, 95, 195401. [CrossRef]

118. Lund, H.E.; Campi, D.; Ruckhofer, A.; Halbritter, S.; Holt, A.J.U.; Bianchi, M.; Bremholm, M.; Benedek, G.; Hofmann, P.; Ernst, W.E.; et al. Electron-Phonon Coupling and (Bulk) Electronic Structure of $\mathrm{Sb}_{2} \mathrm{Te}_{3}$ with intrinsic doping. 2021; To be publish.

119. Heid, R.; Sklyadneva, I.Y.; Chulkov, E.V. Electron-phonon coupling in topological surface states: The role of polar optical modes. Sci. Rep. 2017, 7, 1095. [CrossRef]

120. Chen, C.; Xie, Z.; Feng, Y.; Yi, H.; Liang, A.; He, S.; Mou, D.; He, J.; Peng, Y.; Liu, X.; et al. Tunable Dirac Fermion Dynamics in Topological Insulators. Sci. Rep. 2013, 3, 2411. [CrossRef]

121. Hatch, R.C.; Bianchi, M.; Guan, D.; Bao, S.; Mi, J.; Iversen, B.B.; Nilsson, L.; Hornekær, L.; Hofmann, P. Stability of the $\mathrm{Bi}_{2} \mathrm{Se}_{3}(111)$ topological state: Electron-phonon and electron-defect scattering. Phys. Rev. B 2011, 83, 241303. [CrossRef]

122. Zeljkovic, I.; Scipioni, K.L.; Walkup, D.; Okada, Y.; Zhou, W.; Sankar, R.; Chang, G.; Wang, Y.J.; Lin, H.; Bansil, A.; et al. Nanoscale determination of the mass enhancement factor in the lightly doped bulk insulator lead selenide. Nat. Commun. 2015, 6, 6559. [CrossRef] [PubMed]

123. Campi, D.; Bernasconi, M.; Benedek, G. Ab-initio calculation of surface phonons at the $\mathrm{Sb}_{2} \mathrm{Te}_{3}(111)$ surface. Surf. Sci. 2018, 678, 46-51. [CrossRef]

124. Bussmann, A.; Bilz, H.; Roenspiess, R.; Schwarz, K. Oxygen polarizability in ferroelectric phase transitions. Ferroelectrics 1980, 25, 343-346. [CrossRef]

125. Bussmann-Holder, A.; Benedek, G.; Bilz, H.; Mokross, B. Microscopic polarizability model of ferroelectric soft modes. J. Phys. Colloq. 1981, 42, C6-409-C6-411. [CrossRef]

126. Bilz, H.; Bussmann-Holder, A.; Benedek, G. Ferroelectricity in ternary compounds. Nuovo Cimento D 1983, 2, 1957-1963. [CrossRef]

127. Bilz, H.; Benedek, G.; Bussmann-Holder, A. Theory of ferroelectricity: The polarizability model. Phys. Rev. B 1987, 35, 4840-4849. [CrossRef]

128. Benedek, G.; Bussmann-Holder, A.; Bilz, H. Nonlinear travelling waves in ferroelectrics. Phys. Rev. B 1987, 36, 630-638. [CrossRef]

129. Bussmann-Holder, A.; Bilz, H.; Benedek, G. Applications of the polarizability model to various displacive-type ferroelectric systems. Phys. Rev. B 1989, 39, 9214-9223. [CrossRef]

130. Bilz, H.; Büttner, H.; Bussmann-Holder, A.; Kress, W.; Schröder, U. Nonlinear Lattice Dynamics of Crystals with Structural Phase Transitions. Phys. Rev. Lett. 1982, 48, 264-267. [CrossRef]

131. Bilz, H.; Büttner, H.; Bussmann-Holder, A.; Vogl, P. Phonon anomalies in ferroelectrics and superconductors. Ferroelectrics 1987, 73, 493-500. [CrossRef] 
132. Sigmund, E.; Müller, K.A. (Eds.) Phase Separation in Cuprate Superconductors. In Proceedings of the Second International Workshop on Phase Separation in Cuprate Superconductors, Cottbus, Germany, 4-10 September 1993; Springer Science \& Business Media: Berlin, Germany, 2012.

133. Symmetry and Heterogeneity in High Temperature Superconductors; Bianconi, A. (Ed.) Springer Science \& Business Media: Berlin, Germany, 2006.

134. Shengelaya, A.; Müller, K.A. The intrinsic heterogeneity of superconductivity in the cuprates. Europhys. Lett. 2014, 109, 27001. [CrossRef]

135. Bianconi, A. Resonances and Complexity: From Stripes to Superstripes. J. Supercond. Nov. Magn. 2011, 24, 1117-1121. [CrossRef]

136. Bianconi, A.; Innocenti, D.; Campi, G. Superstripes and Superconductivity in Complex Granular Matter. J. Supercond. Nov. Magn. 2013, 26, 2585-2588. [CrossRef]

137. Bianconi, A. Superstripes in the Low Energy Physics of Complex Quantum Matter at the Mesoscale. J. Supercond. Nov. Magn. 2015, 28, 1227-1229. [CrossRef]

138. Campi, G.; Bianconi, A.; Poccia, N.; Bianconi, G.; Barba, L.; Arrighetti, G.; Innocenti, D.; Karpinski, J.; Zhigadlo, N.D.; Kazakov, S.M.; et al. Inhomogeneity of charge-density-wave order and quenched disorder in a high- $\mathrm{T}_{\mathrm{C}}$ superconductor. Nature 2015, 525, 359-362. [CrossRef]

139. Agrestini, S.; Saini, N.L.; Bianconi, G.; Bianconi, A. The strain of $\mathrm{CuO}_{2}$ lattice: The second variable for the phase diagram of cuprate perovskites. J. Phys. Math. Gen. 2003, 36, 9133-9142. [CrossRef]

140. Jaouen, T.; Hildebrand, B.; Mottas, M.-L.; Di Giovannantonio, M.; Ruffieux, P.; Rumo, M.; Nicholson, C.W.; Razzoli, E.; Barreteau, C.; Ubaldini, A.; et al. Phase separation in the vicinity of Fermi surface hot spots. Phys. Rev. B 2019, 100, 075152. [CrossRef]

141. Bianconi, A.; Poccia, N.; Sboychakov, A.O.; Rakhmanov, A.L.; Kugel, K.I. Intrinsic arrested nanoscale phase separation near a topological Lifshitz transition in strongly correlated two-band metals. Supercond. Sci. Technol. 2015, 28, 024005. [CrossRef]

142. Kostylev, I.; Yonezawa, S.; Wang, Z.; Ando, Y.; Maeno, Y. Uniaxial-strain control of nematic superconductivity in $\mathrm{Sr}_{\mathrm{x}} \mathrm{Bi}_{2} \mathrm{Se}_{3}$. Nat. Commun. 2020, 11, 4152. [CrossRef]

143. Benedek, G.M.; Bernasconi, D.; Campi, I.V.; Silkin, I.P.; Chernov, V.M.; Silkin, E.V.; Chulkov, P.M.; Echenique, J.P.; Toennies, G.; Anemone, A.; et al. Evidence for Acoustic Surface Plasmons from Inelastic Atom Scattering. 2020; Unpublished work.

144. Silkin, V.M.; García-Lekue, A.; Pitarke, J.M.; Chulkov, E.V.; Zaremba, E.; Echenique, P.M. Novel low-energy collective excitation at metal surfaces. Europhys. Lett. 2004, 66, 260. [CrossRef]

145. Shvonski, A.; Kong, J.; Kempa, K. Plasmon-polaron of the topological metallic surface states. Phys. Rev. B 2019, 99, 125148. [CrossRef]

146. Lemmens, L.F.; Devreese, J.T. Collective excitations of the polaron-gas. Solid State Commun. 1974, 14, $1339-1341$. [CrossRef]

147. Mott, N.F. The bipolaron theory of high-temperature superconductors. Phys. Stat. Mech. Appl. 1993, 200, 127-135. [CrossRef]

148. Müller, K.A. Large, Small, and Especially Jahn-Teller Polarons. J. Supercond. 1999, 12, 3-7. [CrossRef]

149. Müller, K.A. The unique properties of superconductivity in cuprates. J. Supercond Nov. Magn. 2014, 27, 2163-2179. [CrossRef]

150. Müller, K.A. Essential Heterogeneities in Hole-Doped Cuprate Superconductors Superconductivity in Complex. Systems: Structure and Bonding; Müller, K.A., Bussmann-Holder, A., Eds.; Springer: Berlin, Germany, 2015; pp. 1-11.

151. Zhao, G.; Conder, K.; Keller, H.; Müller, K.A. Oxygen isotope effects in: Evidence for polaronic charge carriers and their condensation. J. Phys. Condens. Matter 1998, 10, 9055-9066. [CrossRef]

152. Perali, A.; Innocenti, D.; Valletta, A.; Bianconi, A. Anomalous isotope effect near a 2.5 Lifshitz transition in a multi-band multi-condensate superconductor made of a superlattice of stripes. Supercond. Sci. Technol. 2012, 25, 124002. [CrossRef]

153. Lanzara, A.; Zhao, G.; Saini, N.L.; Bianconi, A.; Conder, K.; Keller, H.; Müller, K.A. Oxygen-isotope shift of the charge-stripe ordering temperature in $\mathrm{La}_{2-x} \mathrm{Sr}_{x} \mathrm{CuO}_{4}$ from x-ray absorption spectroscopy. J. Phys. Condens. Matter. 1998, 11, L541. [CrossRef] 
154. Lanzara, A.; Bogdanov, P.V.; Zhou, X.J.; Kellar, S.A.; Feng, D.L.; Lu, E.D.; Yoshida, T.; Eisaki, H.; Fujimori, A.; Kishio, K.; et al. Evidence for ubiquitous strong electron-phonon coupling in high-temperature superconductors. Nature 2011, 412, 510-514. [CrossRef]

155. Benedek, G. The Erice Legacy. Il Nuovo Saggiatore 2019, 35, 47.

Publisher's Note: MDPI stays neutral with regard to jurisdictional claims in published maps and institutional affiliations.

(C) 2020 by the authors. Licensee MDPI, Basel, Switzerland. This article is an open access article distributed under the terms and conditions of the Creative Commons Attribution (CC BY) license (http://creativecommons.org/licenses/by/4.0/). 